\title{
Quantitative Untersuchung der Anhangsorgane der Wangenhaut bei den Japanern."
}

Von

\section{Shisei Kuriki.}

Aus dem Anatomischen Institut der Keio Universität, Tokyo.

Inhalt.

I. Einleitung . . . . . . . . . . . . . . . . . . 584

II. Material und Methode. . . . . . . . . . . . . . . . 584

III. Eigene Befunde . . . . . . . . . . . . . . . . . . . 587

1. Menge der Schweissdrüse . . . . . . . . . . . 587

2. Menge der Talgdrüse . . . . . . . . . . . . . . . . 597

3. Menge des Haarbalgmuskels . . . . . . . . . . . . . 605

IV. Tabellarische Zusammenstellung . . . . . . . . . . . . 613

1. Schweissdrüse. . . . . . . . . . . . . . . 613

a. Menge und Ausmündungšahl der Drüse in der Wangenhaut bei den verschiedenen Altersstufen. . . . . . 613

b. Reihenfolge der Mengengrösse und Ausmündungszahl der Drüse in der Wangenhaut bei den verschiedenen Altersstufen . . . . . . . . . . . . . . 614

2. Talgdrüse . . . . . . . . . . . . . . . . . 615

a. Menge der Drüse und Zahl des Haares in der Wangenhaut bei den verschiedenen Altersstufen. . . . . . . 615

b. Reihenfolge der Mengengröße der Talgdrüse und der Zahl des Haares in der Wangenhaut bei den verschiedenen Altersstufen . . . . . . . . . . . . . . . 616

3. Haarbalgmuskel . . . . . . . . . . . . . . . . . . 617

a. Menge des Haarbalgmuskels und Zahl des Haares in der Wangenhaut bei den verschiedenen Altersstufen. . . . 617

1) Diese Abhandlung bildet die 14. Mitteilung von „Quantitative Untersuchung der Anhangsorgane der Haut von K. Okajima." 
b. Reihenfolge der Mengengrösse des Haarbalgmuskels und der Zahl des Haares in der Wangenhaut bei den verschiedenen Altersstufen . . . . . . . . . . . 618

V. Vergleichungstabellen . . . . . . . . . . . . . . . . .

1. Reihenfolge der Mengengrösse der Schweissdrüse in $1 \mathrm{gcm}$ Hautflächeninhalt an den verschiedenen Körperteilen . . • 619

$\supseteq$ Reihenfolge der Mengengrösse des angenommenen Schweisedrüsenindividuums an den verschiedenen Körperteilen. . . 620

3. Reihenfolge der Ausmündungszahl der Schweissdrïse in $1 \mathrm{gcm}$ Hautflächeninhalt an den verschiedenen Körperteilen . . . 621

4. Reihenfolge der Mengengrösse der Talgdrüse in 1 qcm Hautflächeninhalt an den verschiedenen Körperteilen . . • • . 621

5. Reihenfolge der Mengengrösse des Haarbalgmuskels in $1 \mathrm{gcm}$ Hautflächeninhalt an den verschiedenen Körperteilen . . . 623

VI. Zusammenfassung. . . . . . . . . . . . . . . . . . . 624

\section{Einleitung.}

Wenngleich viele Autoren in unserem Institut die Anbangsorgane der Haut an den verschiedenen Körperregionen bei verschiedenen Rassen, Altersstufen und in beiden Geschlechtern zum Gegenstand der quantitativen Untersuchungen herangezogen haben, ist doch die Wangenhaut bisher von niemandem verfolgt worden; so habe ich in der vorliegenden Arbeit daraufhin eine mengenbestimmende Forschung angestellt.

\section{Material und Methode.}

Für meine Untersuchung, wie in der Tabelle 1 gezeigt, habe ich die Hautstücke der Wange von 10 japanischen Feten und 8 Japanern des verschiedenen Alters verwandt. Die Leichen wurden mit FormolAlkohol-Lösung im ganzen Körper injiziert, und dam in 10\% Formalinlösung aufbewahrt. Die Hautstücke von ca. $1.2 \mathrm{gcm}$ Grösse mit genïgendem Unterhautgewebe wurden sorgfältig mit scharfem Skalpell von der mittleren Wangengegend herausgeschnitten, in Zelloidin eingebettet und in Flächenschnittserien von $40 \mu$ Dicke zerlegt. 
Tabelle 1 .

\begin{tabular}{|c|c|c|c|c|c|}
\hline $\begin{array}{l}\text { Material- } \\
\text { nummer }\end{array}$ & $\begin{array}{l}\text { Leichen- } \\
\text { nunimer }\end{array}$ & Alter & Geschlecht & $\begin{array}{l}\text { Körper- } \\
\text { gewicht }\end{array}$ & Todesursache \\
\hline $\mathbf{I}$ & E.N. 533 & $\begin{array}{c}7 \mathrm{~m} \\
\text { (Fetus) }\end{array}$ & ○ & $0.97 \mathrm{~kg}$ & \\
\hline II & E.N. 488 & $\begin{array}{c}8 \mathrm{~m} \\
\text { (Fetus) }\end{array}$ & $\hat{o}$ & 1.50 & \\
\hline III & E.N. 514 & $\frac{8 \mathrm{~m}}{\text { (Fetus) }}$ & q & 1.50 & \multirow{3}{*}{ 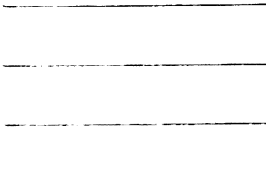 } \\
\hline IV & F.N. 685 & $\begin{array}{c}9 \mathrm{~m} \\
\text { (Fetus) }\end{array}$ & ㅇ & 1.57 & \\
\hline $\mathrm{V}$ & E.N. 569 & $\begin{array}{c}9 \mathrm{~m} \\
\text { (Fetus) }\end{array}$ & $\hat{o}$ & 1.60 & \\
\hline VI & E.N. 456 & $\begin{array}{c}9 \mathrm{~m} \\
\text { (Fetus) }\end{array}$ & ㅇ & 1.70 & \multirow[b]{2}{*}{ 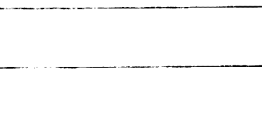 } \\
\hline VII & E.N. 499 & $\begin{array}{c}9 \mathrm{~m} \\
\text { (Fetus) }\end{array}$ & 우 & 2.05 & \\
\hline VIII & E.N. 578 & $\begin{array}{c}9 \mathrm{~m} \\
\text { (Fetus) }\end{array}$ & $\hat{\delta}$ & 2.05 & \\
\hline IX & E.N. 550 & $\begin{array}{c}9 \mathrm{~m} \\
\text { (Fetus) }\end{array}$ & $\hat{o}$ & 2.06 & \\
\hline $\mathbf{X}$ & E.N. 684 & $\begin{array}{c}10 \mathrm{~m} \\
\text { (Fetus) }\end{array}$ & $\hat{\delta}$ & 2.52 & \\
\hline $\mathbf{X I}$ & L.N. 602 & $6 \mathrm{~m}$ & 우 & 2.50 & Herzschwäche \\
\hline *XII & I.N. 447 & $5 \mathrm{~m}$ & ㅇ & 6.80 & $\begin{array}{r}\text { Barlo w'sche } \\
\text { Krankheit }\end{array}$ \\
\hline XIII & I.N. 501 & $3 \mathrm{~m}$ & $\delta$ & 10.68 & Pyothorax \\
\hline XIV & I.N. 506 & $7 \mathrm{j}$ & 우 & 13.60 & unklar \\
\hline $\mathbf{X V}$ & L.N. 589 & $4 \mathrm{~m}$ & $\hat{\delta}$ & 18.00 & Darmtuberkulose \\
\hline XVI & I.N. 360 & $17 \mathrm{j}$ & 우 & 24.83 & Idiotie \\
\hline XVII & I.N. 532 & $14 \mathrm{j}$ & 우 & 27.40 & Lungentuberkulose \\
\hline XVIII & I.N. 499 & $3 \mathrm{~m}$ & $\hat{o}$ & 39.50 & Herzlähmung \\
\hline
\end{tabular}

* Koreanisches Kind.

Die Schnitte wurden mit Hämatoxylin(Hansen)-Eosin oder Karminsäure-Phosphormolybdänlösung nach Okajima gefärbt.

Zur Berechnung des Volumens habe ich folgende Gleichung nach Okajima angewandt:

$$
\mathrm{V}=\frac{\mathrm{FD} \Sigma \mathrm{W}}{\mathrm{W}_{1} \mathrm{~m}^{2}}
$$

$\mathrm{V}=$ Volumen der Gegenstände,

$\mathrm{F}=$ Flächeninhalt des einzelnen Papierstückes,

$\mathrm{W}_{1}=$ Gewicht des einzelnen Papierstückes,

$\mathrm{W}=$ Gewicht des Gegenstandes in einem Schnitt, 


$$
\begin{aligned}
& D=\text { Dicke des Schnittes, } \\
& \mathrm{m}=\text { Vergrösserung. }
\end{aligned}
$$

Die sonstigen Behandlungen nahm ich nach den anderen Autoren vor (s. Literatur).

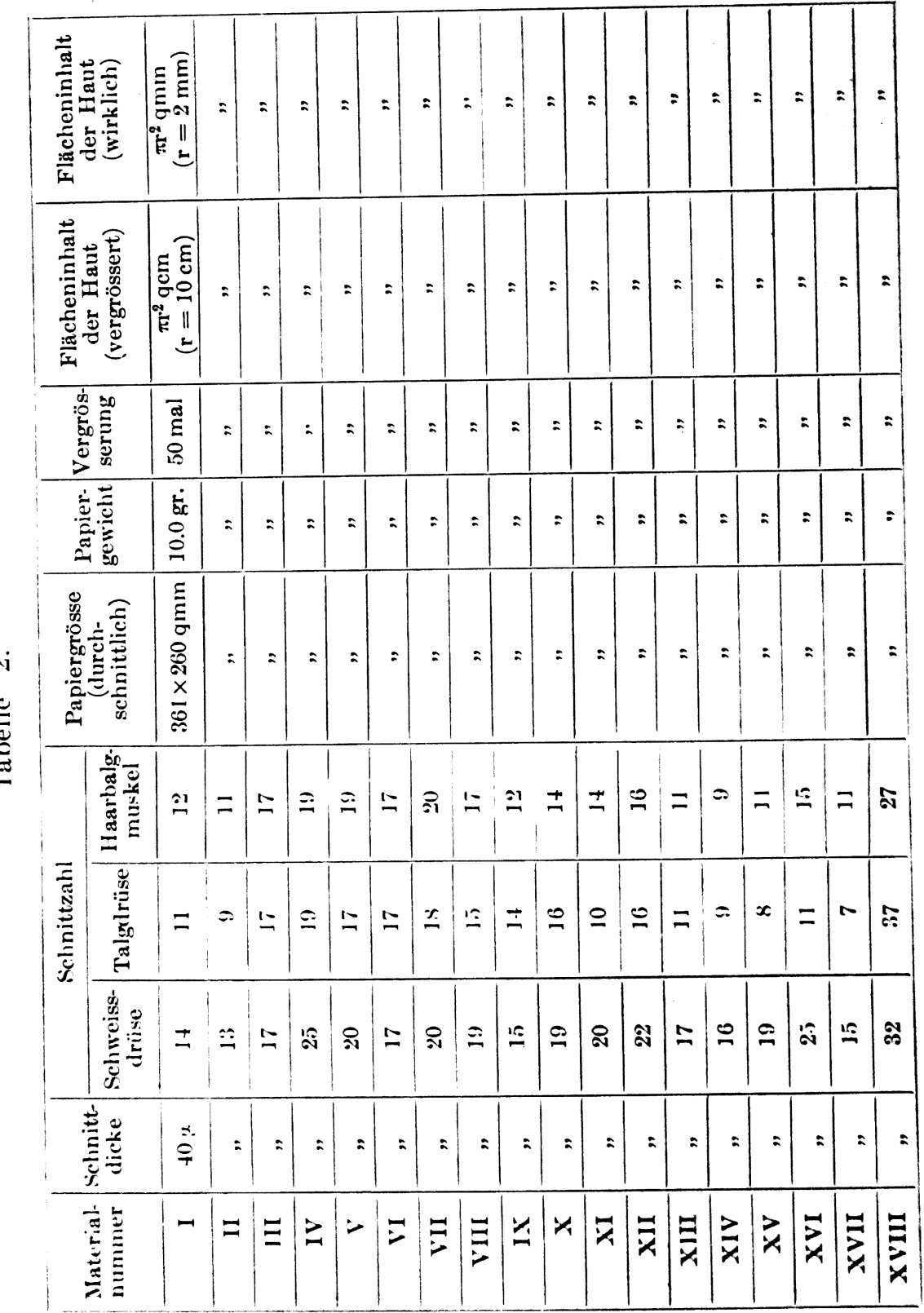




\section{Eigene Befunde.}

\section{Menge der Schweissdrüse.}

\section{Tabelle 3.}

Schweissdrüse. Wangenhaut,

$9 \mathrm{~m}$. Fetus, 우 (E.N. 533).

\begin{tabular}{|c|c|c|}
\hline $\begin{array}{l}\text { Schnitt- } \\
\text { nummer }\end{array}$ & $\begin{array}{c}\text { Zahl der } \\
\text { Drüsen- } \\
\text { stücke }\end{array}$ & $\begin{array}{l}\text { Gewicht der } \\
\text { ausgeschnit- } \\
\text { tenen Papier- } \\
\text { stücke }\end{array}$ \\
\hline 1 & 18 & $7.8 \mathrm{mg}$ \\
\hline 2 & 26 & 14.5 \\
\hline 3 & 31 & 19.0 \\
\hline 4 & 37 & 28.7 \\
\hline 5 & 79 & 66.1 \\
\hline 6 & 96 & 90.6 \\
\hline 7 & 97 & 110.0 \\
\hline 8 & 97 & 122.5 \\
\hline 9 & 85 & 147.8 \\
\hline 10 & 68 & 112.9 \\
\hline 11 & 26 & 18.5 \\
\hline 12 & 16 & 44.9 \\
\hline 13 & 11 & 10.9 \\
\hline \multirow[t]{2}{*}{14} & 3 & 4.0 \\
\hline & & 798.2 \\
\hline
\end{tabular}

Volumen in $\pi \mathrm{r}^{2} \mathrm{qmm}\left(\mathrm{r}=\frac{100}{50} \mathrm{~mm}\right)$

$=\mathrm{V}\left(\right.$ in $\left.\pi \times\left(\frac{10}{5}\right)^{2} \mathrm{gmm}\right)=\frac{\mathrm{FD} \Sigma \mathrm{IV}}{\mathrm{W}_{1} \mathrm{~m}^{2}}$

$=\frac{(361 \times 260) \times 0.04 \times 798.2}{10000 \times 50^{2}}$

$=\frac{29967620.80}{25000000}=0.119870 \mathrm{cmm}$,

also in $1 \mathrm{gcm}$ umgerechnet

$V($ in $1 \mathrm{~g}=\mathrm{m})=0.119870 \times \frac{1}{\pi} \times \frac{2500}{100}$

$=0.119870 \times 0.318309 \times 25$

$=0.953893 \mathrm{cmm}$.

Ferner in $1 \mathrm{gcm}$ gleichmässig verteilt angenommen, bildet die Drüse eine kontinuierliche Schicht in Ausgleichs-(Durchschnitts-)dicke von

$\frac{0.9539}{100}=0.009539 \mathrm{~mm}($ rund $10 \mu)$. 
Tabelle 4.

Schweissdrüse. Wangenhaut, $8 \mathrm{~m}$. Fetus, ô (E.N. 488).

\begin{tabular}{|c|c|c|}
\hline $\begin{array}{l}\text { Schnitt- } \\
\text { nummer }\end{array}$ & $\begin{array}{l}\text { Zahl der } \\
\text { Drüsen- } \\
\text { stücke }\end{array}$ & $\begin{array}{l}\text { Gewicht der } \\
\text { ausgeschnit- } \\
\text { tenen Papier- } \\
\text { stücke }\end{array}$ \\
\hline 1 & 4 & $4.0 \mathrm{mg}$ \\
\hline 2 & 5 & 3.0 \\
\hline 3 & 9 & 5.1 \\
\hline 4 & 16 & 8.0 \\
\hline 5 & 17 & 13.0 \\
\hline 6 & 22 & 27.6 \\
\hline 7 & 36 & 47.0 \\
\hline 8 & 25 & 48.5 \\
\hline 9 & 20 & 39.4 \\
\hline 10 & 15 & 24.0 \\
\hline 11 & 10 & 16.1 \\
\hline 12 & 8 & 15.0 \\
\hline 13 & 3 & 5.9 \\
\hline & & 256.6 \\
\hline
\end{tabular}

$\mathrm{V}$ (in $\left.\pi \mathrm{r}^{2} \mathrm{qmm}\right)=0.038535 \mathrm{cmm}$.

$\mathrm{V}($ in $1 \mathrm{qcm})=0.306651 \mathrm{cmm}$.

Ausgleichsdicke $=0.003067 \mathrm{~mm}$

(rund $3 \mu$.).
Tabelle 5.

Schweissdrüse. Wangenhaut, $8 \mathrm{~m}$. Fetus, 우 (E.N. 514).

\begin{tabular}{|c|c|c|}
\hline $\begin{array}{l}\text { Schnitt- } \\
\text { nummer }\end{array}$ & $\begin{array}{l}\text { Zahl der } \\
\text { Drüsen- } \\
\text { stücke }\end{array}$ & $\begin{array}{l}\text { Gewicht der } \\
\text { ausgeschnit- } \\
\text { tenen Papier- } \\
\text { stücke }\end{array}$ \\
\hline 1 & 21 & $7.4 \mathrm{mg}$ \\
\hline 2 & 23 & 10.0 \\
\hline 3 & 32 & 15.7 \\
\hline 4 & 37 & 16.8 \\
\hline 5 & 55 & 38.0 \\
\hline 6 & 50 & 54.0 \\
\hline 7 & 49 & 43.0 \\
\hline 8 & 47 & 38.5 \\
\hline 9 & 52 & 41.0 \\
\hline 10 & 49 & 38.9 \\
\hline 11 & 42 & 37.8 \\
\hline 12 & 41 & 43.0 \\
\hline 13 & 41 & 45.3 \\
\hline 14 & 32 & 33.5 \\
\hline 15 & 17 & 22.0 \\
\hline 16 & 13 & 13.2 \\
\hline 17 & 6 & 6.5 \\
\hline & & 504.6 \\
\hline
\end{tabular}

$V$ (in $\left.\pi r^{2} q \mathrm{~mm}\right)=0.075779 \mathrm{cmm}$.

$\mathrm{V}$ (in $1 \mathrm{gcm})=0.0603029 \mathrm{cmm}$.

Ausgleichsdicke $=0.006030 \mathrm{~mm}$

(rund $6 \mu$ ). 
Tabelle 6.

Schweissdrüse. Wangenhaut, 9 m. Fetus, 우 (E.N. 685).

\begin{tabular}{|c|c|c|}
\hline $\begin{array}{l}\text { Schnitt- } \\
\text { nummer }\end{array}$ & $\begin{array}{l}\text { Zahl der } \\
\text { Drüsen- } \\
\text { stücke }\end{array}$ & $\begin{array}{c}\text { Gewicht der } \\
\text { ausgeschnit- } \\
\text { tenen Papier- } \\
\text { stücke }\end{array}$ \\
\hline 1 & 8 & $3.0 \mathrm{mg}$ \\
\hline 2 & 11 & 4.7 \\
\hline 3 & 21 & 8.8 \\
\hline 4 & 30 & 13.1 \\
\hline 5 & 36 & 16.0 \\
\hline 6 & 36 & 17.3 \\
\hline 7 & 57 & 31.6 \\
\hline 8 & 54 & 53.4 \\
\hline 9 & 53 & 67.0 \\
\hline 10 & 51 & 48.7 \\
\hline 11 & 46 & 44.8 \\
\hline 12 & 47 & 49.0 \\
\hline 13 & 42 & 36.2 \\
\hline 14 & 36 & 51.6 \\
\hline 15 & 36 & 48.2 \\
\hline 16 & 35 & 55.5 \\
\hline 17 & 30 & 41.7 \\
\hline 18 & 32 & 44.1 \\
\hline 19 & 18 & 34.5 \\
\hline 20 & 18 & 20.3 \\
\hline 21 & 9 & 17.7 \\
\hline 22 & 5 & 7.2 \\
\hline 23 & 4 & 3.9 \\
\hline 24 & 4 & 3.9 \\
\hline 25 & 2 & 1.5 \\
\hline & & 723.7 \\
\hline
\end{tabular}

$\mathrm{V}\left(\right.$ in $\left.\pi \mathrm{r}^{2} \mathrm{gmm}\right)=0.108682 \mathrm{cmm}$.

$\mathrm{V}($ in $1 \mathrm{gcm})=0.864862 \mathrm{cmm}$.

Ausgleichsdicke $=0.008649 \mathrm{~mm}$

(rund $9 \mu$ ).
Tabelle 7.

Schweissdrüse. Wangenhaut, $9 \mathrm{~m}$. Fetus, 占 (E.N. 569).

\begin{tabular}{|c|c|c|}
\hline $\begin{array}{l}\text { Schnitt- } \\
\text { nummer }\end{array}$ & $\begin{array}{l}\text { Zahl der } \\
\text { Drüsen- } \\
\text { stücke }\end{array}$ & $\begin{array}{l}\text { Gewicht der } \\
\text { ausgeschnit- } \\
\text { tenen Papier- } \\
\text { stücke }\end{array}$ \\
\hline 1 & 1 & $0.4 \mathrm{mg}$ \\
\hline 2 & 2 & 1.0 \\
\hline 3 & 4 & 1.5 \\
\hline 4 & 5 & 2.3 \\
\hline 5 & 5 & 3.4 \\
\hline 6 & 9 & 5.5 \\
\hline 7 & 10 & 7.2 \\
\hline 8 & 11 & 11.0 \\
\hline 9 & 21 & 20.2 \\
\hline 10 & 28 & 21.6 \\
\hline 11 & 26 & 32.4 \\
\hline 12 & 27 & 26.0 \\
\hline 13 & 21 & 23.0 \\
\hline 14 & 16 & 18.1 \\
\hline 15 & 14 & 24.5 \\
\hline 16 & 14 & 21.0 \\
\hline 17 & 11 & 12.5 \\
\hline 18 & 6 & 9.0 \\
\hline 19 & 2 & 3.1 \\
\hline 20 & 2 & 0.6 \\
\hline & & 244.3 \\
\hline
\end{tabular}

$\mathrm{V}\left(\right.$ in $\left.\pi \mathrm{r}^{2} \mathrm{qmm}\right)=0.36688 \mathrm{cmm}$.

$\mathrm{V}$ (in $1 \mathrm{gcm})=0.291953 \mathrm{cmm}$.

Ausgleichsdicke $=0.002920 \mathrm{~mm}$ (rund $3 \mu$ ). 
Tabelle 8.

Schweissdrüse. Wangenhaut,

9 m. Fetus, ㅇ (E.N. 456).

\begin{tabular}{|c|c|c|}
\hline $\begin{array}{l}\text { Schnitt- } \\
\text { nummer }\end{array}$ & $\begin{array}{l}\text { Zahl der } \\
\text { Drüsen- } \\
\text { stücke }\end{array}$ & $\begin{array}{c}\text { Gewicht der } \\
\text { ausgeschnit- } \\
\text { tenen Papier- } \\
\text { stücke }\end{array}$ \\
\hline 1 & 11 & $4.6 \mathrm{mg}$ \\
\hline 2 & 22 & 9.0 \\
\hline 3 & 24 & 13.3 \\
\hline 4 & 38 & 29.5 \\
\hline 5 & 55 & 43.0 \\
\hline 6 & 54 & 47.0 \\
\hline 7 & 55 & 43.0 \\
\hline 8 & 61 & 49.0 \\
\hline 9 & 51 & 50.8 \\
\hline 10 & 51 & 45.5 \\
\hline 11 & 41 & 43.1 \\
\hline 12 & 37 & 42.6 \\
\hline 13 & 31 & 34.5 \\
\hline 14 & 14 & 14.2 \\
\hline 15 & 12 & 18.0 \\
\hline 16 & 5 & 8.0 \\
\hline 17 & - & - \\
\hline 18 & - & - \\
\hline 19 & 1 & 2.1 \\
\hline & & 497.2 \\
\hline
\end{tabular}

$\mathrm{V}$ (in $\left.\pi \mathrm{r}^{2} \mathrm{qmm}\right)=0.74668 \mathrm{crnm}$.

$\mathrm{V}($ in $1 \mathrm{gcm})=0.594188 \mathrm{cmm}$.

Ausgleichsdicke $=0.005942 \mathrm{~mm}$

(rund $6 \mu$ ).
Tabelle 9.

Schweissdrüse. . Wangenhaut, $9 \mathrm{~m}$. Fetus, ㅇ (E.N. 499).

\begin{tabular}{|c|c|c|}
\hline $\begin{array}{l}\text { Schnitt- } \\
\text { nummer }\end{array}$ & $\begin{array}{l}\text { Zahl der } \\
\text { Drüsen- } \\
\text { stīcke }\end{array}$ & $\begin{array}{l}\text { Gewicht der } \\
\text { ausgeschnit- } \\
\text { tenen Papier- } \\
\text { stincke }\end{array}$ \\
\hline 1 & 1 & $0.2 \mathrm{mg}$ \\
\hline 2 & 1 & 0.2 \\
\hline 3 & 1 & 0.5 \\
\hline 4 & 1 & 0.8 \\
\hline 5 & 1 & 0.8 \\
\hline 6 & 1 & 0.4 \\
\hline 7 & 2 & 1.1 \\
\hline 8 & 2 & 1.4 \\
\hline 9 & 2 & 5.5 \\
\hline 10 & 5 & 6.0 \\
\hline 11 & 3 & 1.8 \\
\hline 12 & 2 & 2.7 \\
\hline 13 & 4 & 2.7 \\
\hline 14 & 2 & 2.4 \\
\hline 15 & 1 & 0.4 \\
\hline 16 & 1 & 0.4 \\
\hline 17 & - & - \\
\hline 18 & 1 & 0.2 \\
\hline 19 & 1 & 0.4 \\
\hline 20 & 1 & 1.9 \\
\hline 21 & 1 & 6.3 \\
\hline & & 36.1 \\
\hline
\end{tabular}

$V$ (in $\pi \mathrm{r}^{2} \mathrm{qmm}$ ) $=0.005421 \mathrm{cmm}$.

$\mathrm{V}$ (in $1 \mathrm{qcm})=1.182988 \mathrm{cmm}$.

Ausgleichsdicke $=0.011830 \mathrm{~mm}$ 
Quantitative Untersuchung der Anhangsorgane der Wangenhaut bei den Japanern. $\quad 591$

Tabelle 10.

Schweissdrüse. Wangenbaut, $.9 \mathrm{~m}$. Fetus, ô (E.N. 578).

\begin{tabular}{|c|c|c|}
\hline $\begin{array}{l}\text { Schnitt- } \\
\text { nummer }\end{array}$ & $\begin{array}{l}\text { Zahl der } \\
\text { Drüsen- } \\
\text { stücke }\end{array}$ & $\begin{array}{l}\text { Gewicht der } \\
\text { ausgeschnit- } \\
\text { tenen Papier- } \\
\text { stücke }\end{array}$ \\
\hline 1 & 13 & $4.9 \mathrm{mg}$ \\
\hline 2 & 27 & 8.4 \\
\hline 3 & 44 & 14.3 \\
\hline 4 & 66 & 22.2 \\
\hline 5 & 64 & 21.2 \\
\hline 6 & 68 & 21.0 \\
\hline 7 & 71 & 28.3 \\
\hline 8 & 84 & 47.1 \\
\hline 9 & 72 & 70.0 \\
\hline 10 & 95 & 118.1 \\
\hline 11 & 70 & 133.0 \\
\hline 12 & 80 & 164.9 \\
\hline 13 & 68 & 125.1 \\
\hline 14 & 40 & 86.0 \\
\hline 15 & 40 & 48.5 \\
\hline J6 & 24 & 40.0 \\
\hline 17 & 12 & 20.5 \\
\hline 18 & 5 & 11.0 \\
\hline 19 & 2 & 5.4 \\
\hline & : & 989.9 \\
\hline
\end{tabular}

$\mathrm{V}$ (in $\left.\pi \mathrm{r}^{2} \mathrm{gmm}\right)=0.148659 \mathrm{cmm}$.

$\mathrm{V}($ in $1 \mathrm{gcm})=1.182988 \mathrm{cmm}$.

Ausgleichsdicke $=0.011830 \mathrm{~mm}$
Tabelle 11.

Schweissdrüse. Wangenhaut. 9 m. Fetus, ô (E.N. 550).

\begin{tabular}{|c|c|c|}
\hline $\begin{array}{l}\text { Schnitt- } \\
\text { nummer }\end{array}$ & $\begin{array}{l}\text { Zahl der } \\
\text { Drüsen- } \\
\text { stücke }\end{array}$ & $\begin{array}{l}\text { Gewicht der } \\
\text { ausgeschnit- } \\
\text { tenen Papier- } \\
\text { stücke }\end{array}$ \\
\hline 1 & 16 & $6.8 \mathrm{mg}$ \\
\hline 2 & 45 & 19.2 \\
\hline 3 & 80 & 33.8 \\
\hline 4 & 74 & 28.6 \\
\hline 5 & 59 & 27.0 \\
\hline 6 & 48 & 20.4 \\
\hline 7 & 61 & 59.1 \\
\hline 8 & 70 & 89.0 \\
\hline 9 & 59 & 93.0 \\
\hline 10 & 39 & 60.0 \\
\hline 11 & 20 & 20.8 \\
\hline 12 & 6 & 13.3 \\
\hline 13 & 8 & 10.0 \\
\hline 14 & 7 & 12.2 \\
\hline 15 & 1 & 2.4 \\
\hline & & 495.6 \\
\hline
\end{tabular}

$\mathrm{V}\left(\right.$ in $\left.\pi \mathrm{r}^{2} \mathrm{qmm}\right)=0.074427 \mathrm{cmm}$.

$\mathrm{V}($ in $1 \mathrm{gcm})=0.592270 \mathrm{cmm}$.

Ausgleichsdicke $=0.005923 \mathrm{~mm}$

(rund $6 \mu$.).

(rúnd $12 \mu$ ). 
Tabelle 12.

Schweissdrüse. Wangenhaut, $10 \mathrm{in}$. Fetus, of (E.N. 684).

\begin{tabular}{|c|c|c|}
\hline $\begin{array}{l}\text { Schnitt- } \\
\text { nummer }\end{array}$ & $\begin{array}{l}\text { Zahl der } \\
\text { Drüsen- } \\
\text { stücke }\end{array}$ & $\begin{array}{l}\text { Gewicht der } \\
\text { ausgeschnit- } \\
\text { tenen Papier- } \\
\text { stücke }\end{array}$ \\
\hline 1 & 8 & $4.0 \mathrm{mg}$ \\
\hline 2 & 16 & 10.0 \\
\hline 3 & 24 & 19.0 \\
\hline 4 & 27 & 23.5 \\
\hline 5 & 25 & 40.1 \\
\hline 6 & 41 & 61.0 \\
\hline 7 & 41 & 91.9 \\
\hline 8 & 36 & 114.4 \\
\hline 9 & 38 & 149.0 \\
\hline 10 & 28 & 133.0 \\
\hline 11 & 26 & 123.0 \\
\hline 12 & 22 & 107.2 \\
\hline 13 & 24 & 110.5 \\
\hline 14 & 12 & 63.3 \\
\hline 15 & 7 & 29.0 \\
\hline 16 & 6 & 14.0 \\
\hline 17 & 3 & 7.0 \\
\hline 18 & 2 & 5.8 \\
\hline 19 & 1 & 1.5 \\
\hline & & $116,7.2$ \\
\hline
\end{tabular}

$V\left(\right.$ in $\left.\pi \mathrm{r}^{2} \mathrm{qmm}\right)=0.166275 \mathrm{cmm}$.

$V($ in $1 \mathrm{qcm})=1.323172 \mathrm{cmm}$.

Ausgleichsdicke $=0.013232 \mathrm{~mm}$

(rund $13 \mu$ ).
Tabelle 18.

Schweissdrüse. , Wangenbaut, 6 m. Säugling, ㅇ (L.N. 602).

\begin{tabular}{|c|c|c|}
\hline $\begin{array}{l}\text { Schnitt- } \\
\text { nummer }\end{array}$ & $\begin{array}{c}\text { Zahl der } \\
\text { Drüsen- } \\
\text { stücke }\end{array}$ & $\begin{array}{l}\text { Gewicht der } \\
\text { ausgeschnit } \\
\text { tenen Papier- } \\
\text { stücke }\end{array}$ \\
\hline 1 & 4 & $2.8 \mathrm{mg}$ \\
\hline 2 & 5 & 3.0 \\
\hline 3 & 5 & 3.2 \\
\hline 4 & 7 & 5.4 \\
\hline 5 & 9 & 4.9 \\
\hline 6 & 8 & 4.9 \\
\hline 7 & 8 & 10.0 \\
\hline 8 & 8 & 10.5 \\
\hline 9 & 13 & 10.0 \\
\hline 10 & 7 & 29.0 \\
\hline 11 & 6 & 20.0 \\
\hline 12 & 6 & 14.0 \\
\hline 13 & 4 & 11.2 \\
\hline 14 & 5 & 8.0 \\
\hline 15 & 6 & 20.1 \\
\hline 16 & 3 & 21.0 \\
\hline 17 & 2 & 19.4 \\
\hline 18 & 2 & 9.5 \\
\hline 19 & 2 & 11.0 \\
\hline 20 & 1 & 3.1 \\
\hline & & 221.0 \\
\hline
\end{tabular}

$\mathrm{V}$ (in $\left.\pi \mathrm{r}^{2} \mathrm{qmm}\right)=0.033189 \mathrm{cmm}$.

$\mathrm{V}$ (in $1 \mathrm{qcm})=0.264109 \mathrm{cmm}$.

A uggleichedicke $=0.002641 \mathrm{~mm}$ 
Quautitative Untersuchung der Anhangsorgane der Wangenhaut bei den Japanern.

Tabelle 14.

Schweissdrüse. . Wangenhaut, 1 j. u. $5 \mathrm{~m}$. koreanisches Mädchen (L.N. 447).

\begin{tabular}{|c|c|c|}
\hline $\begin{array}{l}\text { Schnitt- } \\
\text { nummer }\end{array}$ & $\begin{array}{l}\text { Zahl der } \\
\text { Drüsen- } \\
\text { stücke }\end{array}$ & $\begin{array}{l}\text { Gewicht der } \\
\text { ausgeschnit- } \\
\text { tenen Papier- } \\
\text { stücke }\end{array}$ \\
\hline 1 & 2 & $1.4 \mathrm{mg}$ \\
\hline 2 & 4 & 29 \\
\hline 3 & 4 & 4.0 \\
\hline 4 & 7 & 7.0 \\
\hline 5 & 10 & 15.0 \\
\hline 6 & 13 & 19.4 \\
\hline 7 & 16 & 29.3 \\
\hline 8 & 19 & 63.0 \\
\hline 9 & 23 & 136.0 \\
\hline 10 & 20 & 172.0 \\
\hline 11 & 21 & 166.5 \\
\hline 12 & 19 & 135.0 \\
\hline 13 & 12 & 80.5 \\
\hline 14 & 11 & 131.0 \\
\hline 15 & 6 & 153.7 \\
\hline 16 & 7 & 133.0 \\
\hline 17 & 9 & 125.2 \\
\hline 18 & 3 & 68.1 \\
\hline 19 & 2 & 53.0 \\
\hline 20 & 2 & 54.0 \\
\hline 21 & 1 & 31.2 \\
\hline 22 & 1 & 29.0 \\
\hline \multicolumn{3}{|r|}{1611.1} \\
\hline
\end{tabular}

$\mathrm{V}$ (in $\left.\pi \mathrm{r}^{2} \mathrm{gmm}\right)=0.241949 \mathrm{cmm}$.

$\mathrm{V}$ (in $1 \mathrm{qcm})=1.925365 \mathrm{cmm}$.

Ausgleichsdicke $=0.019254 \mathrm{~mm}$
Tabelle 15 .

Schweissdrüse. Wangenhaut, 6 j. u. $3 \mathrm{~m}$. o (L.N. 501).

\begin{tabular}{|c|c|c|}
\hline $\begin{array}{l}\text { Schnitt- } \\
\text { nummer }\end{array}$ & $\begin{array}{l}\text { Zahl der } \\
\text { Drüsen- } \\
\text { stücke }\end{array}$ & $\begin{array}{l}\text { Gewicht der } \\
\text { ausgeschnit- } \\
\text { tenen Papier- } \\
\text { stücke }\end{array}$ \\
\hline 1 & 8 & $9.0 \mathrm{mg}$ \\
\hline 2 & 11 & 8.0 \\
\hline 3 & 15 & 9.2 \\
\hline 4 & 17 & 15.7 \\
\hline 5 & 19 & 18.5 \\
\hline 6 & 25 & 96.1 \\
\hline 7 & 31 & 178.5 \\
\hline 8 & 23 & 241.2 \\
\hline 9 & 23 & 252.4 \\
\hline 10 & 16 & 239.0 \\
\hline 11 & 14 & 180.5 \\
\hline 12 & 7 & 167.1 \\
\hline 13 & 3 & 14.9 \\
\hline 14 & 3 & 8.0 \\
\hline 15 & 2 & 0.8 \\
\hline 16 & 2 & 2.2 \\
\hline 17 & 1 & 1.2 \\
\hline & & 1442.3 \\
\hline
\end{tabular}

$\mathrm{V}$ (in $\left.\pi \mathrm{r}^{2} \mathrm{qmm}\right)=0.216599 \mathrm{cmm}$. $\mathrm{V}($ in $1 \mathrm{gcm})=1.723636 \mathrm{cmm}$. Ausgleichsdicke $=0.0172636 \mathrm{~mm}$ (rund $17 \mu$ ). 
Tabelle 16.

Schweissdrüse. Wangenhaut, 7 j. ㅇ (L.N. 506).

\begin{tabular}{|c|c|c|}
\hline $\begin{array}{l}\text { Schnitt- } \\
\text { nummer }\end{array}$ & $\begin{array}{l}\text { Zahl der } \\
\text { Drüsen- } \\
\text { stücke }\end{array}$ & $\begin{array}{l}\text { Gewicht der } \\
\text { ausgeschnit- } \\
\text { tenen Papier- } \\
\text { stücke }\end{array}$ \\
\hline 1 & 20 & $11.1 \mathrm{mg}$ \\
\hline 2 & 20 & 10.2 \\
\hline 3 & 21 & 9.3 \\
\hline 4 & 20 & 9.0 \\
\hline 5 & 23 & 24.2 \\
\hline 6 & 27 & 32.2 \\
\hline 7 & 22 & 85.5 \\
\hline 8 & 25 & 116.0 \\
\hline 9 & 27 & 216.8 \\
\hline 10 & 22 & 350.4 \\
\hline 11 & 17 & 407.0 \\
\hline 12 & 17 & 362.7 \\
\hline 13 & 15 & 261.2 \\
\hline 14 & 10 & 142.7 \\
\hline 15 & 6 & 53.5 \\
\hline 16 & 1 & 31.6 \\
\hline & & 2123.4 \\
\hline
\end{tabular}

$V\left(\right.$ in $\left.\pi \mathrm{r}^{2} \mathrm{qmm}\right)=0.318884 \mathrm{cmm}$.

$\mathrm{V}($ in $1 \mathrm{qcm})=2.537593 \mathrm{cmm}$.

Ausgleichsdicke $=0.025376 \mathrm{~mm}$

(rund $25 \mu$ ).
Tabelle 17.

Schweissdrüse. . Wangenhaut, 8 j. u. 4 m. ô.(L.N. 532).

\begin{tabular}{|c|c|c|}
\hline $\begin{array}{l}\text { Schnitt- } \\
\text { nummer }\end{array}$ & $\begin{array}{l}\text { Zahl der } \\
\text { Drüsen- } \\
\text { stücke }\end{array}$ & $\begin{array}{l}\text { Gewicht der } \\
\text { ausgeschnit. } \\
\text { tenen Papier- } \\
\text { stücke }\end{array}$ \\
\hline 1 & 2 & $1.2 \mathrm{mg}$ \\
\hline 2 & 7 & 2.0 \\
\hline 3 & 10 & 5.4 \\
\hline 4 & 10 & 4.0 \\
\hline 5 & 16 & 20.0 \\
\hline 6 & 18 & 36.0 \\
\hline 7 & 31 & 50.0 \\
\hline 8 & 26 & 132.9 \\
\hline 9 & 16 & 264.0 \\
\hline 10 & 37 & $\mathbf{3 5 0 . 0}$ \\
\hline 11 & 29 & 451.5 \\
\hline 12 & 26 & 371.7 \\
\hline 13 & 22 & 285.4 \\
\hline 14 & 22 & 204.0 \\
\hline 15 & 18 & 146.9 \\
\hline 16 & 14 & 68.0 \\
\hline 17 & 3 & 22.0 \\
\hline 18 & 2 & 8.0 \\
\hline 19 & 3 & 2.2 \\
\hline & & 2483.2 \\
\hline
\end{tabular}

$V\left(\right.$ in $\left.\pi r^{2} q \mathrm{~mm}\right)=0.372917 \mathrm{cmm}$.

$\mathrm{V}($ in $1 \mathrm{gcm})=2.967573 \mathrm{cmm}$.

Ausgleichsdicke $=0.029676 \mathrm{~mm}$

(rund $30 \mu$ ). 
Quantitative Untersuchung der Anhangsorgane der Wangenhaut bei den Japanern.

Tabelle 18 .

Schweissdrüse. Wangenhaut, $17 \mathrm{j}$. 우 (L.N. 360).

\begin{tabular}{|c|c|c|}
\hline $\begin{array}{l}\text { Schnitt- } \\
\text { nummer }\end{array}$ & $\begin{array}{l}\text { Zahl der } \\
\text { Drüsen- } \\
\text { stücke }\end{array}$ & $\begin{array}{l}\text { Gewicht der } \\
\text { ausgeschnit- } \\
\text { tenen Papier- } \\
\text { stücke }\end{array}$ \\
\hline 1 & 4 & $1.9 \mathrm{mg}$ \\
\hline 2 & 18 & 4.7 \\
\hline 3 & 14 & 4.3 \\
\hline 4 & 13 & 5.0 \\
\hline 5 & 12 & 4.5 \\
\hline 6 & 11 & 4.6 \\
\hline 7 & 9 & 4.0 \\
\hline 8 & 13 & 4.5 \\
\hline 9 & 13 & 5.8 \\
\hline 10 & 15 & 8.2 \\
\hline 11 & 19 & 29.7 \\
\hline 12 & 25 & 42.8 \\
\hline 13 & 26 & 131.1 \\
\hline 14 & 28 & 224.8 \\
\hline 15 & 26 & $270 . \overline{5}$ \\
\hline 16 & 23 & 259.5 \\
\hline 17 & 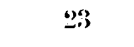 & 241.5 \\
\hline 18 & 21 & 210.7 \\
\hline 19 & 29 & 126.6 \\
\hline 20 & 17 & 111.9 \\
\hline 21 & 6 & 48.0 \\
\hline 22 & 3 & 7.8 \\
\hline & & 1746.4 \\
\hline
\end{tabular}

$\mathrm{V}\left(\right.$ in $\left.\pi \mathrm{r}^{2} \mathrm{gmm}\right)=0.262267 \mathrm{cmm}$.

$\mathrm{V}$ (in $1 \mathrm{gcm})=2.087050 \mathrm{cmm}$.

Ausgleichsdicke $=0.020871 \mathrm{~mm}$
Tabelle 19.

Schweissdrüse. Wangenhaut, 14 j. u. 7 m. ㅇ (L.N. 532).

\begin{tabular}{|c|c|c|}
\hline $\begin{array}{l}\text { Schnitt- } \\
\text { nummer }\end{array}$ & $\begin{array}{l}\text { Zahl der } \\
\text { Drüsen- } \\
\text { stücke }\end{array}$ & $\begin{array}{l}\text { Gewicht der } \\
\text { ausgeschnit- } \\
\text { tenen Papier- } \\
\text { stücke }\end{array}$ \\
\hline 1 & 11 & $5.7 \mathrm{mg}$ \\
\hline 2 & 15) & 6.5 \\
\hline 3 & 16 & 9.0 \\
\hline 4 & 15 & 8.0 \\
\hline 5 & 21 & 13.4 \\
\hline 6 & 2 & 2.8 \\
\hline 7 & 24 & 45.4 \\
\hline 8 & 16 & 85.9 \\
\hline 9 & 24 & 260.0 \\
\hline 10 & 24 & 308.0 \\
\hline 11 & 20 & 395.0 \\
\hline 12 & $2: 2$ & 388.8 \\
\hline 13 & 21 & 198.8 \\
\hline 14 & 9 & 72.9 \\
\hline 15 & 4 & 20.1 \\
\hline & & 1838.3 \\
\hline
\end{tabular}

$\mathrm{V}\left(\right.$ in $\left.\pi \mathrm{r}^{2} \mathrm{qmm}\right)=0.276069 \mathrm{cmm}$.

$\mathrm{V}($ in $1 \mathrm{gcm})=2.196883 \mathrm{cmm}$.

Ausgleichsdicke $=0.021969 \mathrm{~mm}$

(rund $21 \mu$ ). 
Tabelle 20.

Schweissdrüse. Wangenhaut, 40 j. u. 3 m. o (L.N. 499).

\begin{tabular}{|c|c|c|}
\hline $\begin{array}{l}\text { Schnitt- } \\
\text { nummer }\end{array}$ & $\begin{array}{l}\text { Zahl der } \\
\text { Drüsen- } \\
\text { stücke }\end{array}$ & $\begin{array}{l}\text { Gewicht der } \\
\text { ausgeschnit- } \\
\text { tenen Papier- } \\
\text { stücke }\end{array}$ \\
\hline 1 & 4 & $3.0 \mathrm{mg}$ \\
\hline 2 & 5 & 3.8 \\
\hline 3 & 4 & 2.0 \\
\hline 4 & 6 & 2.9 \\
\hline 5 & 6 & 2.3 \\
\hline 6 & 6 & 2.3 \\
\hline 7 & 6 & 2.3 \\
\hline 8 & 6 & 2.0 \\
\hline 9 & 8 & 3.0 \\
\hline 10 & 8 & 3.0 \\
\hline 11 & 10 & 3.7 \\
\hline 12 & 7 & 3.1 \\
\hline 13 & 8 & 5.1 \\
\hline 14 & 8 & 3.5 \\
\hline 15 & 7 & 3.5 \\
\hline 16 & 6 & 9.8 \\
\hline 17 & 6 & 25.4 \\
\hline 18 & 4 & 24.3 \\
\hline 19 & 7 & 37.8 \\
\hline 20 & 9 & 43.0 \\
\hline 21 & 14 & 66.0 \\
\hline 22 & 9 & 120.0 \\
\hline 23 & 9 & 127.9 \\
\hline 24 & 7 & 74.5 \\
\hline 25 & 5 & 77.8 \\
\hline 26 & 5 & 64.5 \\
\hline 27 & 11 & 41.0 \\
\hline 28 & 3 & 21.0 \\
\hline 29 & 3 & 38.8 \\
\hline 30 & 6 & 27.2 \\
\hline 31 & 2 & 17.7 \\
\hline 32 & 1 & 4.8 \\
\hline & & 867.0 \\
\hline
\end{tabular}

$\mathrm{V}$ (in $\left.\pi \mathrm{r}^{2} \mathrm{qmm}\right)=0.130203 \mathrm{cmm}$. $\mathrm{V}($ in $1 \mathrm{qcm})=1.036120 \mathrm{cmm}$. Ausgleichsdicke $=0.010361 \mathrm{~mm}$ (rund $10 \mu$ ). 
Quantitative Untersuchung der Anhangsorgane der Wangenhaut bei den Japanern. $\quad 597$

\section{Menge der Talgdrüse.}

Tabelle 21.

Talgdrüse. Wangenhaut, 7 m. Fetus, 우 (E.N. 533).

\begin{tabular}{|c|c|c|}
\hline $\begin{array}{c}\text { Schnitt- } \\
\text { nummer }\end{array}$ & $\begin{array}{c}\text { Zahl der } \\
\text { Drüsen- } \\
\text { stücke }\end{array}$ & $\begin{array}{c}\text { Gewicht der } \\
\text { ausgeschnit } \\
\text { tenen Papier- } \\
\text { stücke }\end{array}$ \\
\hline 1 & 2 & $3.5 \mathrm{mg}$ \\
2 & 6 & 16.1 \\
3 & 10 & 34.3 \\
4 & 8 & 32.7 \\
5 & 15 & 33.7 \\
6 & 23 & 69.8 \\
7 & 27 & 119.1 \\
8 & 13 & 70.0 \\
9 & 10 & 16.9 \\
10 & 1 & 3.9 \\
11 & 1 & 3.5 \\
\hline & & 403.5 \\
\hline
\end{tabular}

Volumen (in $\left.\pi \mathrm{r}^{2} \mathrm{qmm}\right)=\frac{\mathrm{FD} \Sigma \mathrm{W}}{\mathrm{W}_{1} \mathrm{~m}^{2}}$

$=\frac{(361 \times 260) \times 0.04 \times 403.5}{10000 \times 50^{2}}$

$=0.060596 \mathrm{cmm}$

Volumen (in $1 \mathrm{gcm}$ )

$=0.060596 \times \frac{1}{\pi} \times \frac{2500}{100}$

$=0.482207 \mathrm{cmm}$

Ausgleichsdicke

$=\frac{0.482207}{100}$

$=0.004822 \mathrm{~mm}$ (rund 5 u.).
Tabelle 22.

Talgdrüse. Wangenhaut, $8 \mathrm{~m}$. Fetus, 合 (E.N. 488).

\begin{tabular}{|c|c|c|}
\hline $\begin{array}{c}\text { Schnitt- } \\
\text { nummer }\end{array}$ & $\begin{array}{c}\text { Zahl der } \\
\text { Drüsen- } \\
\text { stücke }\end{array}$ & $\begin{array}{c}\text { Gewicht der } \\
\text { ausgeschnit- } \\
\text { tenen Papier- } \\
\text { stücke }\end{array}$ \\
\hline 1 & 11 & $16.2 \mathrm{mg}$ \\
2 & 17 & 46.5 \\
3 & 22 & 75.2 \\
4 & 31 & 97.0 \\
5 & 27 & 108.9 \\
6 & 17 & 79.1 \\
7 & 10 & 96.8 \\
8 & 10 & 44.0 \\
9 & 3 & 16.4 \\
\hline
\end{tabular}

$\mathrm{V}\left(\right.$ in $\left.\pi \mathrm{r}^{2} \mathrm{qmm}\right)=0.087117 \mathrm{cmm}$.

$\mathrm{V}$ (in $1 \mathrm{gcm})=0.693254 \mathrm{cmm}$.

Ausgleichsdicke $=0.006933 \mathrm{~mm}$ 
Tabelle 23.

Talgdrüse. Wangenhaut, $8 \mathrm{~m}$. Fetus, 우 (E.N. 514).

\begin{tabular}{|c|c|c|}
\hline $\begin{array}{l}\text { Schnitt- } \\
\text { nummer }\end{array}$ & $\begin{array}{l}\text { Zuhl der } \\
\text { Drïsen- } \\
\text { stücke }\end{array}$ & $\begin{array}{l}\text { Gewicht der } \\
\text { ausgeschnit- } \\
\text { tenen Papier- } \\
\text { stücke }\end{array}$ \\
\hline 1 & 4 & $6.6 \mathrm{mg}$ \\
\hline 2 & 6 & 20.2 \\
\hline 3 & 17 & 75.3 \\
\hline 4 & 22 & 158.2 \\
\hline 5 & 42 & 223.0 \\
\hline 6 & 27 & 215.8 \\
\hline 7 & 39 & 261.3 \\
\hline 8 & 33 & 246.0 \\
\hline 9 & 32 & 271.4 \\
\hline 10 & 33 & 234.9 \\
\hline 11 & 33 & 194.5 \\
\hline 12 & 22 & 194.0 \\
\hline 13 & 18 & 224.9 \\
\hline 14 & 16 & 160.8 \\
\hline 15 & 7 & 105.7 \\
\hline 16 & 6 & 43.6 \\
\hline 17 & 2 & 2.5 \\
\hline & & 2638.7 \\
\hline
\end{tabular}

$\mathrm{V}\left(\right.$ in $\left.\pi \mathrm{r}^{2} \mathrm{qmm}\right)=0.396269 \mathrm{cmm}$.

$V($ in $1 \mathrm{gcm})=3.153407 \mathrm{cmm}$.

Ausgleichsdicke $=0.031534 \mathrm{~mm}$

(rund $31 \mu$ ).
Tabelle 24.

Talgdrüse. Wangeuhaut, 9 m. Fetus, 우 (E.N. 685).

\begin{tabular}{|c|c|c|}
\hline $\begin{array}{l}\text { Schnitt- } \\
\text { nummer }\end{array}$ & $\begin{array}{l}\text { Zahl der } \\
\text { Drüsen- } \\
\text { stücke }\end{array}$ & $\begin{array}{l}\text { Gewicht der } \\
\text { ausgeschnit- } \\
\text { tenen Papier- } \\
\text { stũcke }\end{array}$ \\
\hline 1 & 2 & $3.4 \mathrm{mg}$ \\
\hline 2 & 3 & 22.9 \\
\hline 3 & 12 & 71.0 \\
\hline 4 & 12 & 93.2 \\
\hline 5 & 14 & 95.9 \\
\hline 6 & 13 & 98.8 \\
\hline 7 & 14 & 89.9 \\
\hline 8 & 13 & 131.0 \\
\hline 9 & 14 & 119.0 \\
\hline 10 & 13 & 79.4 \\
\hline 11 & 10 & 83.4 \\
\hline 12 & 10 & 75.3 \\
\hline 13 & 9 & 80.3 \\
\hline 14 & 11 & 83.4 \\
\hline 15 & 12 & 88.1 \\
\hline 16 & 8 & 55.0 \\
\hline 17 & 8 & 48.9 \\
\hline 18 & 8 & 38.4 \\
\hline 19 & 5 & 15.5 \\
\hline & & 1372.8 \\
\hline
\end{tabular}

$V\left(\right.$ in $\left.\pi r^{2} g \mathrm{~mm}\right)=0.206162 \mathrm{cmm}$.

$\mathrm{V}($ in $1 \mathrm{qcm})=1.640582 \mathrm{cmm}$.

Ausgleichsdicke $=0.016406 \mathrm{~mm}$

(rund 16 $\mu$ ). 
Quanțitative Untersuchung der Anhangsorgane der Wangenhaut bei den Japanern. 599

Tabelle 25.

Talgdrüse. Wangenhaut, $9 \mathrm{~m}$. Fetus, 오 (E.N. 569).

\begin{tabular}{|c|c|c|}
\hline $\begin{array}{l}\text { Schnitt- } \\
\text { nummer }\end{array}$ & $\begin{array}{c}\text { Zahl der } \\
\text { Drüsen- } \\
\text { stücke }\end{array}$ & $\begin{array}{l}\text { Gewicht der } \\
\text { ausgeschnit- } \\
\text { tenen Papier- } \\
\text { stücke }\end{array}$ \\
\hline 1 & 8 & $12.4 \mathrm{mg}$ \\
\hline 2 & 17 & 59.7 \\
\hline 3 & 18 & 73.8 \\
\hline 4 & 38 & 135.8 \\
\hline 5 & 40 & 176.0 \\
\hline 6 & 40 & 185.7 \\
\hline 7 & 41 & 195.5 \\
\hline 8 & 38 & 204.0 \\
\hline 9 & 34 & 185.0 \\
\hline 10 & 33 & 209.5 \\
\hline 11 & 20 & 139.2 \\
\hline 12 & 18 & 118.2 \\
\hline 13 & 10 & 61.8 \\
\hline 14 & 6 & 52.0 \\
\hline 15 & 4 & 17.9 \\
\hline 16 & 3 & 22.8 \\
\hline 17 & 2 & 9.1 \\
\hline & & 1858.4 \\
\hline
\end{tabular}

$\mathrm{V}\left(\right.$ in $\left.\pi \mathrm{r}^{2} \mathrm{gmm}\right)=0.279087 \mathrm{cmm}$.

$\mathrm{V}($ in $1 \mathrm{qcm})=2.220899 \mathrm{cmm}$.

Ausgleichsdicke $=0.022209 \mathrm{~mm}$
Tabelle 26.

Talgdrüse. Wangenhaut, 9 m. Fetus, ㅇ (E.N. 456).

\begin{tabular}{|c|c|c|}
\hline $\begin{array}{l}\text { Schnitt. } \\
\text { nummer }\end{array}$ & $\begin{array}{l}\text { Zahl der } \\
\text { Drüsen- } \\
\text { stücke }\end{array}$ & $\begin{array}{c}\text { Gewicht der } \\
\text { ausgeschnit- } \\
\text { tenen Papier- } \\
\text { stiucke }\end{array}$ \\
\hline 1 & 3 & $2.7 \mathrm{mg}$ \\
\hline 2 & 16 & 19.0 \\
\hline 3 & 39 & 57.9 \\
\hline 4 & 28 & 104.1 \\
\hline 5 & 45 & 95.3 \\
\hline 6 & 37 & 114.6 \\
\hline 7 & 37 & 99.8 \\
\hline 8 & 35 & 78.7 \\
\hline 9 & 26 & 87.6 \\
\hline 10 & 43 & 85.4 \\
\hline 11 & 31 & 100.7 \\
\hline 12 & 20 & 51.4 \\
\hline 13 & 15 & 39.6 \\
\hline 14 & 10 & 33.6 \\
\hline 15 & 6 & 24.0 \\
\hline 16 & 7 & 17.7 \\
\hline 17 & 7 & 9.3 \\
\hline & & 1021.4 \\
\hline
\end{tabular}

$\mathrm{V}\left(\right.$ in $\left.\pi \mathrm{r}^{2} \mathrm{gmm}\right)=0.153390 \mathrm{cmm}$.

$\mathrm{V}($ in $1 \mathrm{qcm})=1.220636 \mathrm{cmm}$.

Ausgleichsdicke $=0.012206 \mathrm{~mm}$

(rund $12 \mu$ ). 
Tabelle 27.

Talgdrüse. Wangenhaut, 9 m. Fetus, ㅇ (E.N. 499).

\begin{tabular}{|c|c|c|}
\hline $\begin{array}{l}\text { Schnitt- } \\
\text { nummer }\end{array}$ & $\begin{array}{l}\text { Zahl der } \\
\text { Drïsen- } \\
\text { stïcke }\end{array}$ & $\begin{array}{c}\text { Gewicht der } \\
\text { susgeschnit- } \\
\text { tenen Papier- } \\
\text { stücke }\end{array}$ \\
\hline 1 & 2 & $2.0 \mathrm{mg}$ \\
\hline 2 & 6 & 5.1 \\
\hline 3 & 9 & 36.8 \\
\hline 4 & 23 & 64.0 \\
\hline 5 & 35 & 123.4 \\
\hline 6 & 37 & 205.0 \\
\hline 7 & 42 & 292.6 \\
\hline 8 & 51 & 293.5 \\
\hline 9 & 45 & 281.5 \\
\hline 10 & 42 & 310.9 \\
\hline 11 & 38 & 251.6 \\
\hline 12 & 30 & 181.5 \\
\hline 13 & 33 & 169.8 \\
\hline 14 & 14 & 132.7 \\
\hline 15 & 15 & 104.0 \\
\hline 16 & 12 & 66.9 \\
\hline 17 & 8 & 20.0 \\
\hline \multirow[t]{2}{*}{18} & 1 & 1.0 \\
\hline & & 2541.7 \\
\hline
\end{tabular}

$\mathrm{V}$ (in $\left.\pi \mathrm{r}^{2} \mathrm{qmm}\right)=0.381702 \mathrm{cmm}$.

$\mathrm{V}$ (in $1 \mathrm{qcm})=3.037481 \mathrm{cmm}$.

Ausgleichsdicke $=0.030375 \mathrm{~mm}$

(rund $30 \mu$ ).
Tabelle 28.

Talgdrüse. Wangenhaut, 9 m. Fetus, oิ (E.N. 578).

\begin{tabular}{|c|c|c|}
\hline $\begin{array}{l}\text { Schnitt- } \\
\text { nummer }\end{array}$ & $\begin{array}{l}\text { Zahl der } \\
\text { Drüsen- } \\
\text { stücke }\end{array}$ & $\begin{array}{l}\text { Gewicht der } \\
\text { ausqeashalt. } \\
\text { tenen Papier } \\
\text { stücke }\end{array}$ \\
\hline 1 & 5 & $21.7 \mathrm{mg}$ \\
\hline 2 & 13 & 149.3 \\
\hline 3 & 15 & 264.6 \\
\hline 4 & 26 & 370.0 \\
\hline 5 & 19 & 505.1 \\
\hline 6 & 40 & 366.0 \\
\hline 7 & 31 & 533.0 \\
\hline 8 & 26 & 285.0 \\
\hline 9 & 17 & 157.1 \\
\hline 10 & 10 & 110.0 \\
\hline 11 & 7 & 103.0 \\
\hline 12 & 6 & 37.9 \\
\hline & & 2902.7 \\
\hline
\end{tabular}

$\mathrm{V}\left(\right.$ in $\left.\pi \mathrm{r}^{2} \mathrm{gmm}\right)=0.135053 \mathrm{cmm}$. $\mathrm{V}$ (in $1 \mathrm{qcm})=1.074715 \mathrm{cmm}$. Ausgleichsdicke $=0.010747 \mathrm{~mm}$ (rund $11 \mu$ ). 
Quantitative Untersuchung der Anhangsorgane der Wangenhaut bei den Japanern. 601

Tabelle 29.

Talgdrŭse. Wangenhaut, $9 \mathrm{~m}$. Fetus, ô (E.N: 550).

\begin{tabular}{|c|c|c|}
\hline $\begin{array}{l}\text { Schnitt- } \\
\text { nummer }\end{array}$ & $\begin{array}{l}\text { Zahl der } \\
\text { Drüsen. } \\
\text { stücke }\end{array}$ & $\begin{array}{c}\text { Gewicht der } \\
\text { ausgeschnit- } \\
\text { tenen Papier- } \\
\text { stücke }\end{array}$ \\
\hline 1 & 1 & $1.2 \mathrm{mg}$ \\
\hline 2 & 10 & 12.0 \\
\hline 3 & 31 & 112.4 \\
\hline 4 & 60 & 502.5 \\
\hline 5 & 43 & 773.7 \\
\hline 6 & 56 & 961.6 \\
\hline 7 & 52 & 906.9 \\
\hline 8 & 45 & 822.1 \\
\hline 9 & 41 & 634.9 \\
\hline 10 & 39 & 406.5 \\
\hline 11 & 24 & 218.8 \\
\hline 12 & 15 & 132.0 \\
\hline 13 & 4 & 30.0 \\
\hline 14 & 5 & 28.0 \\
\hline & & 5542.6 \\
\hline
\end{tabular}

$\mathrm{V}\left(\right.$ in $\left.\pi \mathrm{r}^{2} \mathrm{qmm}\right)=0.832365 \mathrm{cmm}$.

$\mathrm{V}($ in $1 \mathrm{qcm})=6.623736 \mathrm{cmm}$.

Ausgleichsdicke $=0.066237 \mathrm{~mm}$

(rund $66 \mu$ ).
Tabelle 30.

Talgdrüse. Wangenhaut, 10 m. Fetus, ô (E.N. 684).

\begin{tabular}{|c|c|c|}
\hline $\begin{array}{l}\text { Schnitt- } \\
\text { nummer }\end{array}$ & $\begin{array}{l}\text { Zahl der } \\
\text { Drüsen- } \\
\text { stücke }\end{array}$ & $\begin{array}{l}\text { Gewicht der } \\
\text { ausgeschnit- } \\
\text { tenen Papier- } \\
\text { stücke }\end{array}$ \\
\hline 1 & 4 & $28.1 \mathrm{mg}$ \\
\hline 2 & 18 & 129.4 \\
\hline 3 & 34 & 323.8 \\
\hline 4 & 33 & 540.4 \\
\hline 5 & 38 & 776.5 \\
\hline 6 & 34 & 828.3 \\
\hline 7 & 33 & 731.8 \\
\hline 8 & 33 & 709.5 \\
\hline 9 & 24 & 554.4 \\
\hline 10 & 19 & 369.0 \\
\hline 11 & 11 & 227.5 \\
\hline 12 & 9 & 133.6 \\
\hline 13 & 5 & 59.4 \\
\hline 14 & 4 & 29.4 \\
\hline 15 & 2 & 17.0 \\
\hline & & 5458.1 \\
\hline
\end{tabular}

$\mathrm{V}\left(\right.$ in $\left.\pi \mathrm{r}^{2} \mathrm{gmm}\right)=0.819676 \mathrm{cmm}$.

$\mathrm{V}$ (in $1 \mathrm{gcm})=6.522760 \mathrm{cmm}$.

Ausgleichsdicke $=0.065228 \mathrm{~mm}$ 
Tabelle 31.

Talgdrüse. Waugenhaut, $6 \mathrm{~m}$. 우 (L.N. 602).

\begin{tabular}{|c|c|c|}
\hline $\begin{array}{c}\text { Schnitt- } \\
\text { nummer }\end{array}$ & $\begin{array}{c}\text { Zahl der } \\
\text { Drüsen- } \\
\text { stücke }\end{array}$ & $\begin{array}{c}\text { Gewicht der } \\
\text { ausgeschnit- } \\
\text { tenen Papier- } \\
\text { stücke }\end{array}$ \\
\hline & 1 & $0.9 \mathrm{mg}$ \\
1 & 1.5 \\
2 & 4 & 8.4 \\
3 & 12 & 24.0 \\
4 & 15 & 43.0 \\
5 & 25 & 78.2 \\
6 & 33 & 80.5 \\
7 & 33 & 83.5 \\
8 & 18 & 24.5 \\
9 & 6 & 8.3 \\
10 & 4 & 352.8 \\
\hline
\end{tabular}

$V\left(\right.$ in $\left.\pi \mathrm{r}^{2} \mathrm{qmm}\right)=0.052982 \mathrm{cmm}$.

$V($ in $1 \mathrm{gcm})=0.421616 \mathrm{cmm}$.

Ausgleichsdicke $=0.004216 \mathrm{~mm}$ (rund $4 \mu$ ).

Tabelle 32 .

Talgdrüse. Wangenhaut, $1 \mathrm{j}$. u. $5 \mathrm{~m}$. 우 (L.N. 447).

\begin{tabular}{|c|c|c|}
\hline $\begin{array}{c}\text { Schnitt- } \\
\text { nummer }\end{array}$ & $\begin{array}{c}\text { Zahl der } \\
\text { Drüsen- } \\
\text { stücke }\end{array}$ & $\begin{array}{c}\text { Gewicht der } \\
\text { ausgeschnit- } \\
\text { tenen Papier- } \\
\text { stücke }\end{array}$ \\
\hline & & $6.8 \mathrm{mg}$ \\
1 & 4 & 16.0 \\
2 & 7 & 29.8 \\
3 & 7 & 56.1 \\
4 & 7 & 67.1 \\
5 & 11 & 124.5 \\
6 & 12 & 104.0 \\
7 & 13 & 56.7 \\
8 & 9 & 38.0 \\
9 & 3 & 63.3 \\
10 & 8 & 89.3 \\
11 & 5 & 56.0 \\
1.2 & 7 & 19.5 \\
13 & 3 & 13.0 \\
14 & 1 & 2.0 \\
15 & 1 & 13.0 \\
16 & 1 & \\
\hline
\end{tabular}

$V$ (in $\left.\pi \mathrm{r}^{2} \mathrm{qmm}\right)=0.113400 \mathrm{cmm}$

$V$ (in $1 \mathrm{qcm})=0.9024085 \mathrm{cmm}$.

Ausgleichsdicke $=0.090241 \mathrm{~mm}$

(rund $90 \mu)$.
Tabelle 33,

Talgdrüse. Wangeuhaut,

6 j. u. $3 \mathrm{~m}$. o (L.N. 501).

\begin{tabular}{|c|c|c|}
\hline $\begin{array}{c}\text { Schnitt. } \\
\text { nummer }\end{array}$ & $\begin{array}{c}\text { Zahl der } \\
\text { Drüsen- } \\
\text { stücke }\end{array}$ & $\begin{array}{c}\text { Gewicht der } \\
\text { ausgeschnit- } \\
\text { tenen Papier- } \\
\text { stingke }\end{array}$ \\
\hline 1 & 5 & $5.8 \mathrm{mg}$ \\
2 & 22 & 54.0 \\
3 & 22 & 141.0 \\
4 & 19 & 182.3 \\
5 & 20 & 242.0 \\
6 & 12 & 102.0 \\
7 & 10 & 51.7 \\
8 & 3 & 53.6 \\
9 & 4 & 53.5 \\
10 & 4 & 13.4 \\
\hline
\end{tabular}

$V$ (in $\left.\pi \mathrm{r}^{2} \mathrm{qmm}\right)=0.135053 \mathrm{cmm}$.

$\mathrm{V}$ (in $1 \mathrm{gcm}$ ) $=1.074715 \mathrm{cmm}$.

Ausgleichsdicke $=0.010747 \mathrm{~nm}$

(rund $11 \mu$ ).

Tabelle 34.

Talgdrüse. Wangenhaut, 7 j. 우 (L.N. 506).

\begin{tabular}{|c|c|c|}
\hline $\begin{array}{c}\text { Schnitt. } \\
\text { nummer }\end{array}$ & $\begin{array}{c}\text { Zahl der } \\
\text { Drüsen- } \\
\text { stücke }\end{array}$ & $\begin{array}{c}\text { Gewicht der } \\
\text { ausgeschnit- } \\
\text { tenen Papier- } \\
\text { stincke }\end{array}$ \\
\hline 1 & 5 & $5.5 \mathrm{mg}$ \\
2 & 13 & 37.0 \\
3 & 10 & 51.1 \\
4 & 12 & 61.0 \\
5 & 17 & 144.4 \\
6 & 10 & 180.9 \\
7 & 11 & 103.6 \\
8 & 3 & 32.2 \\
9 & 4 & 16.4 \\
\hline
\end{tabular}

$V$ (in $\pi \mathrm{r}^{2} \mathrm{qmm}$ ) $=0.094926 \mathrm{cmm}$. $\mathrm{V}$ (in $1 \mathrm{qcin})=0.755395 \mathrm{cmm}$.

Ausgleich sdicke $=0.007554 \mathrm{~mm}$

(rund $8 \mu$ ). 
Quantitative Untersuchung der Anhangsorgane der Wangenhaut bei den Japanern. 603

Tabelle 35.

Talgdrüse. Wangenhaut,

8 j. u. 4 m. oิ (L.N. 589).

\begin{tabular}{|c|c|c|}
\hline $\begin{array}{c}\text { Schnitt- } \\
\text { nummer }\end{array}$ & $\begin{array}{c}\text { Zahl der } \\
\text { Drüisen- } \\
\text { stücke }\end{array}$ & $\begin{array}{c}\text { Gewicht der } \\
\text { ausgeschnit- } \\
\text { tenen Papier- } \\
\text { stücke }\end{array}$ \\
\hline 1 & 3 & $5.3 \mathrm{mg}$ \\
2 & 2 & 6.8 \\
3 & 19 & 70.0 \\
4 & 17 & 91.0 \\
5 & 8 & 55.2 \\
6 & 7 & 63.0 \\
7 & 4 & 38.5 \\
8 & 1 & 3.5 \\
\hline
\end{tabular}

$\mathrm{V}$ (in $\left.\pi \mathrm{r}^{2} \mathrm{gmm}\right)=0.050054 \mathrm{cmm}$.

$\mathrm{V}$ (in $1 \mathrm{qcm})=0.398322 \mathrm{cmm}$.

Ausgleichsdicke $=0.003983 \mathrm{~mm}$

(rund $4 \mu$.).

Tabelle 36.

Talgdrüse. Wangenhaut, 17 j. ㅇ (L.N. 360).

\begin{tabular}{|c|c|c|}
\hline $\begin{array}{c}\text { Schnitt- } \\
\text { nummer }\end{array}$ & $\begin{array}{c}\text { Zahl der } \\
\text { Drüsen- } \\
\text { stücke }\end{array}$ & $\begin{array}{c}\text { Gewicht der } \\
\text { ausgeschnit- } \\
\text { tenen Papier- } \\
\text { stiicke }\end{array}$ \\
\hline 1 & 1 & $0.5 \mathrm{mg}$ \\
2 & 6 & 7.0 \\
3 & 9 & 6.0 \\
4 & 18 & 8.0 \\
5 & 17 & 14.7 \\
6 & 11 & 19.0 \\
7 & 12 & 27.9 \\
8 & 9 & 31.0 \\
9 & 8 & 35.4 \\
10 & 4 & 15.0 \\
11 & 2 & 7.0 \\
\hline
\end{tabular}

$\mathrm{V}$ (in $\left.\pi \mathrm{r}^{2} \mathrm{gmm}\right)=0.025756 \mathrm{cmm}$.

$\mathrm{V}$ (in $1 \mathrm{gcm})=0.204959 \mathrm{cmm}$.

Ausgleichsdicke $=0.002050 \mathrm{~mm}$

(rund $2 \mu$. ).
Tabelle 37.

Talgdrüse. Wangenlaaut, 14 j. u. 7 m. 우 (L.N. 532).

\begin{tabular}{|c|c|c|}
\hline $\begin{array}{c}\text { Schnitt. } \\
\text { nummer }\end{array}$ & $\begin{array}{c}\text { Zahl der } \\
\text { Drüsen- } \\
\text { stücke }\end{array}$ & $\begin{array}{c}\text { Gewicht der } \\
\text { ausgeschnit- } \\
\text { tenen Papier- } \\
\text { stücke }\end{array}$ \\
\hline 1 & 1 & $1.2 \mathrm{mg}$ \\
2 & 8 & 14.5 \\
3 & 6 & 10.3 \\
4 & 9 & 67.5 \\
5 & 16 & 137.8 \\
6 & 9 & 78.5 \\
7 & 7 & 39.0 \\
\hline
\end{tabular}

$\mathrm{V}$ (in $\left.\pi \mathrm{r}^{2} \mathrm{qmm}\right)=0.052306 \mathrm{cmm}$.

$\mathrm{V}$ (in $1 \mathrm{qcm})=0.416237 \mathrm{cmm}$.

Ausgleichsdicke $=0.0041624 \mathrm{~mm}$

(rund $4 \mu$ ). 
Tabelle 38.

Talgdrüse. Wangenhaut, 40 j. u. $3 \mathrm{~m}$. o (L.N. 499).

\begin{tabular}{|c|c|c|}
\hline $\begin{array}{l}\text { Schnitt- } \\
\text { nummer }\end{array}$ & $\begin{array}{l}\text { Zahl der } \\
\text { Drüsen- } \\
\text { stücke }\end{array}$ & $\begin{array}{l}\text { Gewicht der } \\
\text { ausgesehnit- } \\
\text { tenen Papier- } \\
\text { stücke }\end{array}$ \\
\hline $\begin{array}{r}1 \\
2 \\
3 \\
4 \\
5 \\
6 \\
7 \\
8 \\
9 \\
10 \\
11 \\
12 \\
13 \\
14 \\
15 \\
16 \\
17 \\
18 \\
19 \\
20 \\
21 \\
22 \\
23 \\
24 \\
25 \\
26 \\
27 \\
28 \\
29 \\
30 \\
31 \\
32 \\
33 \\
34 \\
35 \\
36 \\
37\end{array}$ & $\begin{array}{r}13 \\
9 \\
6 \\
7 \\
5 \\
7 \\
7 \\
8 \\
16 \\
19 \\
20 \\
27 \\
29 \\
30 \\
37 \\
36 \\
37 \\
32 \\
28 \\
26 \\
30 \\
23 \\
30 \\
19 \\
24 \\
14 \\
14 \\
11 \\
9 \\
6 \\
3 \\
5 \\
4 \\
3 \\
2 \\
2 \\
1\end{array}$ & $\begin{array}{r}6.2 \mathrm{mg} \\
144 \\
24.0 \\
33.0 \\
54.4 \\
55.7 \\
53.0 \\
77.3 \\
170.0 \\
209.3 \\
300.8 \\
383.0 \\
505.8 \\
752.0 \\
907.9 \\
874.5 \\
965.5 \\
996.0 \\
989.9 \\
930.1 \\
965.5 \\
838.0 \\
\mathbf{7 8 9 . 8} \\
698.2 \\
600.7 \\
503.1 \\
\mathbf{4 8 8 . 0} \\
382.0 \\
299.0 \\
222.1 \\
78.8 \\
99.0 \\
\mathbf{8 5 . 0} \\
55.7 \\
47.8 \\
30.5 \\
6.0 \\
\end{array}$ \\
\hline & & 14492.0 \\
\hline
\end{tabular}

$V\left(\right.$ in $\left.\pi \mathrm{r}^{2} \mathrm{gmm}\right)=2.176351 \mathrm{cmm}$.

$\mathrm{V}$ (in $1 \mathrm{qcm})=17.318806 \mathrm{cmm}$.

Ausgleichsdicke $=0.173188 \mathrm{~mm}$

(rund $173 \mu$ ). 
Quantitative Untersuchung der Anhangsorgane der Wangenhaut bei den Japanern. $\quad 605$

\section{Menge des Haarbalgmuskels.}

Tabeile 39.

Haarbalgmuskel. Wangenhaut, 7 m. Fetus, 우 (E.N. 533).

\begin{tabular}{|c|c|c|}
\hline $\begin{array}{c}\text { Schnitt- } \\
\text { nummer }\end{array}$ & $\begin{array}{c}\text { Zahl der } \\
\text { Muskel- } \\
\text { stücke }\end{array}$ & $\begin{array}{c}\text { Gewicht der } \\
\text { ausgeschnit- } \\
\text { tenen Papier- } \\
\text { stücke }\end{array}$ \\
\hline 1 & 10 & $28.8 \mathrm{mg}$ \\
2 & 14 & 37.8 \\
3 & 18 & 35.3 \\
4 & 15 & 34.2 \\
5 & 26 & 64.2 \\
6 & 18 & 38.6 \\
7 & 33 & 102.6 \\
8 & 35 & 134.3 \\
9 & 18 & 60.0 \\
10 & 8 & 19.5 \\
11 & 7 & 30.8 \\
12 & 2 & 11.4 \\
\hline & & 597.5 \\
\hline
\end{tabular}

$\mathrm{V}\left(\right.$ in $\left.\pi \mathrm{r}^{2} \mathrm{qmm}\right)=\frac{\mathrm{FD} \Sigma \mathrm{W}}{\mathrm{W}_{1} \mathrm{~m}^{2}}$

$=\frac{(361 \times 260) \times 0.04 \times 597.5}{10000 \times 50^{2}}$

$=0.089730 \mathrm{cmm}$

$\mathrm{V}$ (in $1 \mathrm{gcm}$ )

$=0.089730 \times \frac{1}{\pi} \times \frac{2500}{100}$

$=0.714047 \mathrm{cmm}$

Ausgleichsdicke

$=0.007140 \mathrm{~mm}$ (rund $7 \mu$.).
Tabelle 40.

Haarbalgmuskel. Wangenhaut, 8 m. Fetus, ô (E.N. 488).

\begin{tabular}{|c|c|c|}
\hline $\begin{array}{c}\text { Schnitt- } \\
\text { nummer }\end{array}$ & $\begin{array}{c}\text { Zahl der } \\
\text { Muskel- } \\
\text { stücke }\end{array}$ & $\begin{array}{c}\text { Gewicht der } \\
\text { ausgeschnit- } \\
\text { tenen Papier- } \\
\text { stücke }\end{array}$ \\
\hline 1 & 8 & $12.0 \mathrm{mg}$ \\
2 & 36 & 41.0 \\
3 & 48 & 42.6 \\
4 & 36 & 74.8 \\
5 & 33 & 54.9 \\
6 & 39 & 68.1 \\
7 & 26 & 62.3 \\
8 & 29 & 89.4 \\
9 & 27 & 71.9 \\
10 & 19 & 54.2 \\
11 & 4 & 16.0 \\
\hline & & 587.2 \\
\hline
\end{tabular}

$\mathrm{V}$ (in $\left.\pi \mathrm{r}^{2} \mathrm{qmm}\right)=0.088183 \mathrm{cmm}$.

$\mathrm{V}($ in $1 \mathrm{gcm})=0.701732 \mathrm{cmm}$.

Ausgleichsdicke $=0.007017 \mathrm{~mm}$

(rund $7 \mu$ ). 
Tabelle 41.

Haarbalgmuskel. Wangenhaut, $8 \mathrm{~m}$. Fetus, 우 (E.N. 514).

\begin{tabular}{|c|c|c|}
\hline $\begin{array}{l}\text { Schnitt- } \\
\text { nummer }\end{array}$ & $\begin{array}{l}\text { Zahl der } \\
\text { Muskel- } \\
\text { stücke }\end{array}$ & $\begin{array}{l}\text { Gewicht der } \\
\text { ausgeschnit- } \\
\text { tenen Papier- } \\
\text { stücke }\end{array}$ \\
\hline 1 & 5 & $6.7 \mathrm{mg}$ \\
\hline 2 & - & - \\
\hline 3 & 1 & 2.6 \\
\hline 4 & 19 & 35.7 \\
\hline 5 & 16 & 30.6 \\
\hline 6 & 26 & 70.0 \\
\hline 7 & 24 & 54.3 \\
\hline 8 & 41 & 91.8 \\
\hline 9 & 39 & 86.1 \\
\hline 10 & 10 & 21.1 \\
\hline 11 & 22 & 53.4 \\
\hline 12 & 18 & 37.5 \\
\hline 13 & 17 & 42.9 \\
\hline 14 & 8 & 27.8 \\
\hline 15 & 8 & 18.5 \\
\hline 16 & 3 & 6.4 \\
\hline 17 & 3 & $: 3.1$ \\
\hline 18 & 1 & 3.0 \\
\hline & & 593.5 \\
\hline
\end{tabular}

$V\left(\right.$ in $\left.\pi \mathrm{r}^{2} \mathrm{qmm}\right)=0.089129 \mathrm{cmm}$.

$\mathrm{V}$ (in $1 \mathrm{gcm})=0.709265 \mathrm{cmm}$.

Ausgleichsdicke $=0.007093 \mathrm{~mm}$

(rund $7 \mu$ ).
Tabelle 42.

Haarbalgmuskel. Wangenhaut, 9 m. Fetus, ㅇ (E.N. 685).

\begin{tabular}{|c|c|c|}
\hline $\begin{array}{l}\text { Schnitt- } \\
\text { nummer }\end{array}$ & $\begin{array}{l}\text { Zahl der } \\
\text { Muskel- } \\
\text { stïcke }\end{array}$ & $\begin{array}{l}\text { Gewicht dor } \\
\text { augeechnit } \\
\text { tenen Papier } \\
\text { stîcke }\end{array}$ \\
\hline 1 & 7 & $47.7 \mathrm{mg}$ \\
\hline 2 & 14 & 61.2 \\
\hline 3 & 15 & 85.1 \\
\hline 4 & 17 & 124.5 \\
\hline 5 & 17 & 130.1 \\
\hline 6 & 15 & 92.2 \\
\hline 7 & 14 & 62.1 \\
\hline 8 & 16 & 52.5 \\
\hline 9 & 13 & 98.5 \\
\hline 10 & 14 & 49.5 \\
\hline 11 & 20 & 53.8 \\
\hline 12 & 18 & 29.9 \\
\hline 13 & 16 & 51.5 \\
\hline 14 & 18 & 44.7 \\
\hline 15 & 11 & 23.3 \\
\hline 16 & 10 & 28.6 \\
\hline 17 & 5 & 10.3 \\
\hline 18 & 9 & 14.8 \\
\hline 19 & 5 & 3.0 \\
\hline
\end{tabular}

$V$ (in $\left.\pi \mathrm{r}^{2} \mathrm{qmm}\right)=0.158931 \mathrm{cmm}$.

$V($ in $1 \mathrm{qcm})=1.264730 \mathrm{cmm}$.

Ausgleichsdicke $=0.012647 \mathrm{~mm}$ 
Tabelle 43.

Haarbalgmuskel. Wangenhaut, 9 m. Fetus, o (E.N. 569).

\begin{tabular}{|c|c|c|}
\hline $\begin{array}{l}\text { Schnittr } \\
\text { nummer }\end{array}$ & $\begin{array}{l}\text { Zahl der } \\
\text { Muskel- } \\
\text { stücke }\end{array}$ & $\begin{array}{l}\text { Gewicht der } \\
\text { ausgeschnit- } \\
\text { tenen Papier- } \\
\text { stücke }\end{array}$ \\
\hline 1 & 8 & $11.0 \mathrm{mg}$ \\
\hline 2 & 1 & 4.6 \\
\hline 3 & 8 & 25.4 \\
\hline 4 & 24 & 40.0 \\
\hline 5 & 20 & 57.5 \\
\hline 6 & 25 & 50.3 \\
\hline 7 & 40 & 112.5 \\
\hline 8 & 33 & 106.3 \\
\hline 9 & 47 & 129.0 \\
\hline 10 & 45 & 174.7 \\
\hline 11 & 34 & 138.9 \\
\hline 12 & 35 & 133.0 \\
\hline 13 & 25 & 92.5 \\
\hline 14 & 31 & 107.9 \\
\hline 15 & 16 & 54.1 \\
\hline 16 & 13 & 46.7 \\
\hline 17 & 9 & 29.1 \\
\hline 18 & 3 & 5.6 \\
\hline 19 & - & - \\
\hline 20 & - & - \\
\hline 21 & 2 & 3.5 \\
\hline & & 1325.1 \\
\hline
\end{tabular}

$V$ (in $\pi r^{2} \mathrm{qmm}$ ) $=0.198998 \mathrm{cmm}$.

$\mathrm{V}($ in $1 \mathrm{gcm})=1.583572 \mathrm{cmm}$.

Ausgleichsdicke $=0.015835 \mathrm{~mm}$

(rund $16 \mu$ ).
Tabelle 44.

Haarbalgmuskel. Wangenhaut, 9 m. Fetus, 우 (E.N. 456).

\begin{tabular}{|c|c|c|}
\hline $\begin{array}{l}\text { Schnitt- } \\
\text { nummer }\end{array}$ & $\begin{array}{l}\text { Zahl der } \\
\text { Muskel- } \\
\text { stücke }\end{array}$ & $\begin{array}{l}\text { Gewicht der } \\
\text { ausgeschnit- } \\
\text { tenen Papier- } \\
\text { stücke }\end{array}$ \\
\hline 1 & 9 & $15.2 \mathrm{mg}$ \\
\hline 2 & 5 & 6.5 \\
\hline 3 & 7 & 14.0 \\
\hline 4 & 32 & 47.4 \\
\hline 5 & 25 & 59.0 \\
\hline 6 & 23 & 46.3 \\
\hline 7 & 30 & 83.8 \\
\hline 8 & 43 & 82.5 \\
\hline 9 & 31 & 72.0 \\
\hline 10 & 19 & 27.0 \\
\hline 11 & 32 & 47.5 \\
\hline 12 & 12 & 28.5 \\
\hline 13 & 11 & 23.5 \\
\hline 14 & 4 & 7.1 \\
\hline 15 & 3 & 1.5 \\
\hline 16 & 5 & 6.9 \\
\hline 17 & 2 & 1.0 \\
\hline & & 569.7 \\
\hline
\end{tabular}

$\mathrm{V}$ (in $\left.\pi \mathrm{r}^{2} \mathrm{gmm}\right)=0.085555 \mathrm{cmm}$.

$\mathrm{V}($ in $1 \mathrm{gcm})=0.680824 \mathrm{cmm}$.

Ausgleichsdicke $=0.006808 \mathrm{~mm}$

(rund $7 \mu)$. 
Tabelle 45.

Haarbalgmuskel. Wangenhaut, 9 m. Fetus, ㅇ (E.N. 499).

\begin{tabular}{|c|c|c|}
\hline $\begin{array}{l}\text { Schnitt- } \\
\text { nummer }\end{array}$ & $\begin{array}{c}\text { Zahl der } \\
\text { Muskel- } \\
\text { stiicke }\end{array}$ & $\begin{array}{l}\text { Gewicht der } \\
\text { ausgeschnit- } \\
\text { tenen Papier- } \\
\text { stücke }\end{array}$ \\
\hline 1 & 2 & $1.1 \mathrm{mg}$ \\
\hline 2 & 5 & 1.8 \\
\hline 3 & 13 & 57.3 \\
\hline 4 & 22 & 82.9 \\
\hline 5 & 27 & 83.0 \\
\hline 6 & 49 & 208.2 \\
\hline 7 & 45 & 206.0 \\
\hline 8 & 43 & 259.3 \\
\hline 9 & 43 & 254.1 \\
\hline 10 & 60 & 278.8 \\
\hline 11 & 38 & 177.7 \\
\hline 12 & 48 & 230.9 \\
\hline 13 & 49 & 175.0 \\
\hline 14 & 29 & 149.8 \\
\hline 15 & 31 & 168.5 \\
\hline 16 & 20 & 82.9 \\
\hline 17 & 14 & 38.6 \\
\hline 18 & 10 & 46.0 \\
\hline 19 & 9 & 19.9 \\
\hline 20 & 4 & 6.0 \\
\hline 21 & 1 & 2.0 \\
\hline 22 & 1 & 0.4 \\
\hline & & 2530.2 \\
\hline
\end{tabular}

$V\left(\right.$ in $\left.\pi r^{2} \mathrm{qmm}\right)=0.379975 \mathrm{cmm}$.

$\mathrm{V}($ in $1 \mathrm{gcm})=3.023738 \mathrm{cmm}$.

Ausgleichsdicke $=0.030237 \mathrm{~mm}$

(rund $30 \mu$ ).
Tabelle 46.

Haurbalgmnokel. Wancenhavt,

9 m. Fotus, oิ (E.N. 578).

\begin{tabular}{|c|c|c|}
\hline $\begin{array}{c}\text { Schnitt- } \\
\text { nummer }\end{array}$ & $\begin{array}{c}\text { Zahl der } \\
\text { Muskel- } \\
\text { stücke }\end{array}$ & $\begin{array}{c}\text { Gewicht der } \\
\text { ausgeschnit- } \\
\text { tenen Papier- } \\
\text { stücke }\end{array}$ \\
\hline & 5 & $5.8 \mathrm{mg}$ \\
1 & 52 & 54.0 \\
2 & 22 & 141.0 \\
3 & 19 & 182.3 \\
4 & 20 & 242.0 \\
5 & 12 & 102.0 \\
6 & 10 & 51.7 \\
7 & 3 & 53.6 \\
8 & 4 & 58.5 \\
9 & 4 & 13.4 \\
10 & & \\
\hline & & 899.3 \\
\hline
\end{tabular}

$\mathrm{V}$ (in $\left.\pi \mathrm{r}^{2} \mathrm{qmm}\right)=0.435916 \mathrm{cmm}$.

$\mathrm{V}$ (in $1 \mathrm{qcm})=3.464890 \mathrm{cmm}$.

Ausgleichsdicke $=0.034649 \mathrm{~mm}$

(rund $35 \mu$ ).

Tabelle 47.

Haarbalgmuskel. Wangenhaut, 9 m. Fetus, oิ (E.N. 550).

\begin{tabular}{|c|c|c|}
\hline $\begin{array}{c}\text { Schnitt- } \\
\text { nummer }\end{array}$ & $\begin{array}{c}\text { 7ahl der } \\
\text { Muskel- } \\
\text { stïcke }\end{array}$ & $\begin{array}{c}\text { Gewicht der } \\
\text { ausgeschnit- } \\
\text { tenen Papler- } \\
\text { stiicke }\end{array}$ \\
\hline & & $65.9 \mathrm{mg}$ \\
1 & 20 & 150.0 \\
2 & 45 & 135.0 \\
3 & 32 & 157.0 \\
4 & 44 & 35.0 \\
5 & 20 & 33.0 \\
6 & 17 & 38.0 \\
7 & 14 & 71.0 \\
8 & 28 & 94.5 \\
9 & 27 & 63.2 \\
10 & 20 & 90.5 \\
11 & 16 & 55.0 \\
12 & 12 & \\
\hline
\end{tabular}

$V$ (in $\left.\pi \mathrm{r}^{2} \mathrm{qmm}\right)=0.148389 \mathrm{cmm}$. $V($ in $1 \mathrm{qcm})=1.180840 \mathrm{cmm}$.

Ausgleichsdicke $=0.011808 \mathrm{~mm}$

(rund 12 $\mu$ ). 
Quantitative Untersuchung der Anhangaorgane der Wangenhaut bei den Japanern.

Tabelle 48.

Haarbalgmuskel. Wangenbaut, 10 m. Fetus, o. (E.N. 684).

\begin{tabular}{|c|c|c|}
\hline $\begin{array}{l}\text { Schnitt- } \\
\text { nummer }\end{array}$ & $\begin{array}{l}\text { Zahl der } \\
\text { Muskel- } \\
\text { stücke }\end{array}$ & $\begin{array}{l}\text { Gewicht der } \\
\text { ausgeschnit- } \\
\text { tenen Papier- } \\
\text { stücke }\end{array}$ \\
\hline 1 & 2 & $5.1 \mathrm{mg}$ \\
\hline 2 & 15 & 182.7 \\
\hline 3 & 25 & 149.0 \\
\hline 4 & 36 & 269.2 \\
\hline 5 & 42 & 219.4 \\
\hline 6 & 31 & 149.4 \\
\hline 7 & 33 & 189.9 \\
\hline 8 & 17 & 103.0 \\
\hline 9 & 18 & 67.5 \\
\hline 10 & 9 & 42.7 \\
\hline 11 & 4 & 14.3 \\
\hline 12 & 3 & 5.3 \\
\hline 13 & 1 . & 5.1 \\
\hline 14 & . 2 & 10.1 \\
\hline & & 1412.7 \\
\hline
\end{tabular}

$V$ (in $\left.\pi r^{2} \mathrm{gmm}\right)=0.212154 \mathrm{cmm}$.

$\mathrm{V}($ in $1 \mathrm{gcm})=1.688264 \mathrm{cmm}$.

Ausgleichsdicke $=0.016883 \mathrm{~mm}$

(rund 17 \%).
Tabelle 49.

Haarbalgmuskel. Wangenbaut, . 6 m. ㅇ (L.N. 602).

\begin{tabular}{|c|c|c|}
\hline $\begin{array}{l}\text { Sehnitt- } \\
\text { nummer }\end{array}$ & $\begin{array}{c}\text { Zahl der } \\
\text { Muskel- } \\
\text { stücke }\end{array}$ & $\begin{array}{l}\text { Gewicht der } \\
\text { ausgeschnit- } \\
\text { tenen Papier- } \\
\text { stücke }\end{array}$ \\
\hline 1 & 5 & $8.0 \mathrm{mg}$ \\
\hline 2 & 4 & 11.3 \\
\hline 3 & 12 & 27.5 \\
\hline 4 & 22 & 47.3 \\
\hline 5 & 27 & 62.5 \\
\hline 6 & 51 & 110.0 \\
\hline 7 & 39 & 152.0 \\
\hline 8 & 44 & 115.0 \\
\hline 9 & 50 & 114.3 \\
\hline 10 & 51 & 164.2 \\
\hline 11 & 56 & 204.5 \\
\hline 12 & 49 & 107.0 \\
\hline 13 & 36 & 84.0 \\
\hline 14 & 14 & 67.5 \\
\hline 15 & 9 & 30.1 \\
\hline & & 1305.2 \\
\hline
\end{tabular}

$\mathrm{V}$ (in $\left.\pi \mathrm{r}^{2} \mathrm{qmm}\right)=0.196010 \mathrm{cmm}$.

$\mathrm{V}($ in $1 \mathrm{gcm})=1.559803 \mathrm{cmm}$.

Ausgleichsdicke $=0.015598 \mathrm{~mm}$

(rund $16 \mu$ ). 
Tabelle 50.

Haarbalgmuskel. Wangenhaut, 1 j. u. 5 m. ㅇ (L.N. 447).

\begin{tabular}{|c|c|c|}
\hline $\begin{array}{l}\text { Sehmitt- } \\
\text { nummer }\end{array}$ & $\begin{array}{l}\text { Zahl der } \\
\text { Muskel- } \\
\text { stïcke }\end{array}$ & $\begin{array}{l}\text { Gewicht der } \\
\text { ausgeschnit- } \\
\text { tenen Papier- } \\
\text { stïcke }\end{array}$ \\
\hline 1 & 1 & $1.3 \mathrm{mg}$ \\
\hline$\ddot{2}$ & - & - \\
\hline 3 & 6 & 86.3 \\
\hline 4 & 7 & 138.7 \\
\hline 5 & 19 & 187.2 \\
\hline (j) & $1 s$ & $6 \because .2$ \\
\hline 7 & ¡) & 107.5 \\
\hline 8 & 7 & 67.0 \\
\hline 9 & 7 & 123.9 \\
\hline 10 & 8 & $\pi \pi .8$ \\
\hline 11 & 7 & 64.2 \\
\hline 12 & 9 & 92.9 \\
\hline 13 & $\therefore$ & 43.0 \\
\hline 14 & 9 & 44.5 \\
\hline 1.i & 2 & 6.0 \\
\hline 16 & $\ddot{\prime}$ & 17.0 \\
\hline & & 1167.5 \\
\hline
\end{tabular}

$V\left(\right.$ in $-r^{2}(\mathrm{gmm})=0.175334 \mathrm{cmm}$.

$\mathrm{V}$ (in 1 (fom) $=1.395261 \mathrm{cmm}$.

Ausgleichsclicke $-0.013953 \mathrm{~mm}$

(rimd 14!).
Tabelle 51.

Haarbalgmuskel. Wangenbaut, 6 j. u. 3 m. o (L.N. 501).

\begin{tabular}{|c|c|c|}
\hline $\begin{array}{c}\text { Schnitt- } \\
\text { nummer }\end{array}$ & $\begin{array}{c}\text { Zahl der } \\
\text { Muskel- } \\
\text { stücke }\end{array}$ & $\begin{array}{c}\text { Gewicht der } \\
\text { ausgeschnit- } \\
\text { tenen Prpier- } \\
\text { stücke }\end{array}$ \\
\hline 1 & 5 & $21.7 \mathrm{mg}$ \\
2 & 13 & 149.3 \\
3 & 15 & 204.6 \\
4 & 26 & 370.0 \\
5 & 19 & 505.1 \\
6 & 40 & 366.1 \\
7 & 31 & 533.0 \\
8 & 26 & 285.0 \\
9 & 17 & 157.1 \\
10 & 10 & 110.0 \\
11 & 7 & 103.0 \\
12 & 6 & 37.9 \\
\hline & & \\
\hline
\end{tabular}

$V$ (in $\left.\pi \mathrm{r}^{2} \mathrm{qmm}\right)=0.435916 \mathrm{cmm}$. $V$ (in $1 \mathrm{qcm}$ ) $=3.464890 \mathrm{cmm}$.

Ausgleichsdicke $=0.034649 \mathrm{~mm}$ (rund $35 \mu$ ).

Tabelle 52.

Haarbalgnuuskel. Wangenhaut, 7 j., ? (L.N. .506).

\begin{tabular}{|c|c|c|}
\hline $\begin{array}{c}\text { Schnitt- } \\
\text { nummer }\end{array}$ & $\begin{array}{c}\text { Zahl der } \\
\text { Iuskel. } \\
\text { stücke }\end{array}$ & $\begin{array}{c}\text { Gewicht ler } \\
\text { ausgeschnit- } \\
\text { tenen Papier- } \\
\text { stücke }\end{array}$ \\
\hline 1 & 1 & $18.5 \mathrm{mg}$ \\
2 & $!$ & 154.0 \\
$: 3$ & 11 & 203.4 \\
4 & 17 & 327.2 \\
5 & 11 & 281.5 \\
6 & 12 & 476.0 \\
7 & $!$ & 497.3 \\
8 & 9 & 379.9 \\
$! 1$ & 5 & 299.0 \\
\hline & & \\
\hline
\end{tabular}

$V$ (in $\left.\pi r^{2} q \mathrm{~mm}\right)=0.411002 \mathrm{cmm}$. $V$ (in $1 \mathrm{qcm})=3.270643 \mathrm{cmm}$.

Ausgleichsdicke $=0.032706 \mathrm{~mm}$ (rund $33 \mu$ ). 
Tabelle 53.

Haarbalgmuskel. Wangenhaut, 8 j. u. 4 m. ô (L.N. 589).

\begin{tabular}{|c|c|c|}
\hline $\begin{array}{l}\text { Schnitt- } \\
\text { nummer }\end{array}$ & $\begin{array}{l}\text { Zahl der } \\
\text { Muskel- } \\
\text { stücke }\end{array}$ & $\begin{array}{c}\text { Gewicht der } \\
\text { ausgeschnit- } \\
\text { tenen Papier- } \\
\text { stücke }\end{array}$ \\
\hline 1 & 1 & $1.0 \mathrm{mg}$ \\
\hline 2 & 8 & 25.0 \\
\hline 3 & 15 & 98.8 \\
\hline 4 & 24 & 104.0 \\
\hline 5 & 35 & 173.5 \\
\hline 6 & 30 & 254.8 \\
\hline 7 & 21 & 312.0 \\
\hline 8 & 20 & 198.7 \\
\hline 9 & 14 & 173.4 \\
\hline 10 & 7 & 146.2 \\
\hline 11 & 4 & 65.0 \\
\hline & & 1552.4 \\
\hline
\end{tabular}

$\mathrm{V}\left(\right.$ in $\left.\pi \mathrm{r}^{2} \mathrm{qmm}\right)=0.233133 \mathrm{cmm}$.

$\mathrm{V}($ in $1 \mathrm{gcm})=1.855210 \mathrm{cmm}$.

Ausgleichsdicke $=0.018552 \mathrm{~mm}$

(rund $19 \mu$ ).
Tabelle 54 .

Haarbalgmuskel. Wangenhaut, 17 j. ㅇ (I.N. 360).

\begin{tabular}{|c|c|c|}
\hline $\begin{array}{l}\text { Schnitt- } \\
\text { nummer }\end{array}$ & $\begin{array}{l}\text { Zah] der } \\
\text { Muskel- } \\
\text { stücke }\end{array}$ & $\begin{array}{l}\text { Gewicht der } \\
\text { ausgeschnit- } \\
\text { tenen Papier- } \\
\text { stücke }\end{array}$ \\
\hline 1 & 1 & $1.0 \mathrm{mg}$ \\
\hline 2 & 3 & 6.5 \\
\hline 3 & 6 & 11.7 \\
\hline 4 & 11 & 31.0 \\
\hline 5 & 22 & $10 \overline{5} .0$ \\
\hline 6 & 42 & 135.2 \\
\hline 7 & 41 & 246.3 \\
\hline 8 & 49 & 267.0 \\
\hline 9 & 38 & 292.0 \\
\hline 10 & 36 & 313.2 \\
\hline 11 & 26 & 286.1 \\
\hline 12 & 26 & 324.0 \\
\hline 13 & 31 & 378.5 \\
\hline 14 & 19 & 200.0 \\
\hline 15 & 10 & 315.0 \\
\hline 16 & 8 & 96.4 \\
\hline 17 & 5 & 105.0 \\
\hline & & 3113.9 \\
\hline
\end{tabular}

$V\left(\right.$ in $\left.\pi r^{2} q m m\right)=0.467633 \mathrm{cmm}$.

$\mathrm{V}($ in $1 \mathrm{qcm})=3.721297 \mathrm{cmm}$.

Ausgleichsdicke $=0.037213 \mathrm{~mm}$

(rund $37 \mu$ ). 
Tabelle 55.

Haarbalginuskel. Wangenhaut, 14 j. u. 7 m. ㅇ (L.N. 532).

\begin{tabular}{|c|c|c|}
\hline $\begin{array}{c}\text { Schnitt. } \\
\text { nummer }\end{array}$ & $\begin{array}{c}\text { Zahl der } \\
\text { Muskel- } \\
\text { stücke }\end{array}$ & $\begin{array}{c}\text { Gewicht der } \\
\text { ausgeschnit- } \\
\text { tenen Papier- } \\
\text { stücke }\end{array}$ \\
\hline 1 & 3 & $35.0 \mathrm{mg}$ \\
2 & 17 & 281.8 \\
3 & 22 & 342.9 \\
4 & 27 & 504.1 \\
5 & 14 & 560.2 \\
6 & 21 & 621.8 \\
7 & 20 & 185.5 \\
8 & 17 & 172.5 \\
9 & 13 & 246.6 \\
10 & 7 & 172.0 \\
11 & 2 & 35.7 \\
\hline & & 3158.1 \\
\hline & & \\
\hline & & \\
\hline
\end{tabular}

$V\left(\right.$ in $\left.\pi r^{2} q \mathrm{~mm}\right)=0.474271 \mathrm{cmm}$.

$\mathrm{V}($ in $1 \mathrm{gcm})=3.774121 \mathrm{cmm}$.

Ausgleichsclicke $=0.0377121 \mathrm{~mm}$

(rund $38 \mu$ ).
Tabelle 56.

Haarbalgmuskel. Wangenhaut, 40 j. u. $3 \mathrm{~m}$. oิ (L.N. 499).

\begin{tabular}{|c|c|c|}
\hline $\begin{array}{l}\text { Schnitt- } \\
\text { nummer }\end{array}$ & $\begin{array}{l}\text { Zahl der } \\
\text { Muskel- } \\
\text { stücke }\end{array}$ & $\begin{array}{c}\text { Gewicht der } \\
\text { ausgeschnit- } \\
\text { tenen Papier- } \\
\text { stücke }\end{array}$ \\
\hline 1 & 2 & $4.2 \mathrm{mg}$ \\
\hline 2 & 3 & 7.4 \\
\hline 3 & 3 & 11.2 \\
\hline 4 & 7 & 66.3 \\
\hline 5 & 1 & 2.4 \\
\hline 6 & 6 & 47.1 \\
\hline 7 & 7 & 60.0 \\
\hline 8 & 5 & 45.1 \\
\hline 9 & 14 & 105.0 \\
\hline 10 & 19 & 149.4 \\
\hline 11 & 18 & 93.0 \\
\hline 12 & 21 & 148.0 \\
\hline 13 & 16 & 86.1 \\
\hline 14 & 21 & 123.0 \\
\hline 15 & 13 & 85.3 \\
\hline 16 & 17 & 89.4 \\
\hline 17 & 17 & 65.3 \\
\hline 18 & 9 & 34.4 \\
\hline 19 & 9 & 32.5 \\
\hline 20 & 9 & 50.0 \\
\hline 21 & 7 & 49.5 \\
\hline 22 & 7 & 61.9 \\
\hline 23 & 9 & 56.0 \\
\hline 24 & 10 & 65.3 \\
\hline 25 & 7 & 38.2 \\
\hline 26 & 6 & 57.0 \\
\hline \multirow[t]{2}{*}{27} & 4 & 20.0 \\
\hline & & 1653.0 \\
\hline
\end{tabular}

$V$ (in $\left.\pi r^{2} \mathrm{qmm}\right)=0.248241 \mathrm{cmm}$.

$\mathrm{V}$ (in $1 \mathrm{gcm})=1.975435 \mathrm{cmm}$.

Ausgleichsdicke $=0.019754 \mathrm{~mm}$

(rund $20 \mu$ ). 
Quantitative Untersuchung der Anhangsorgane der Wangenhaut bei den Japanern.

613

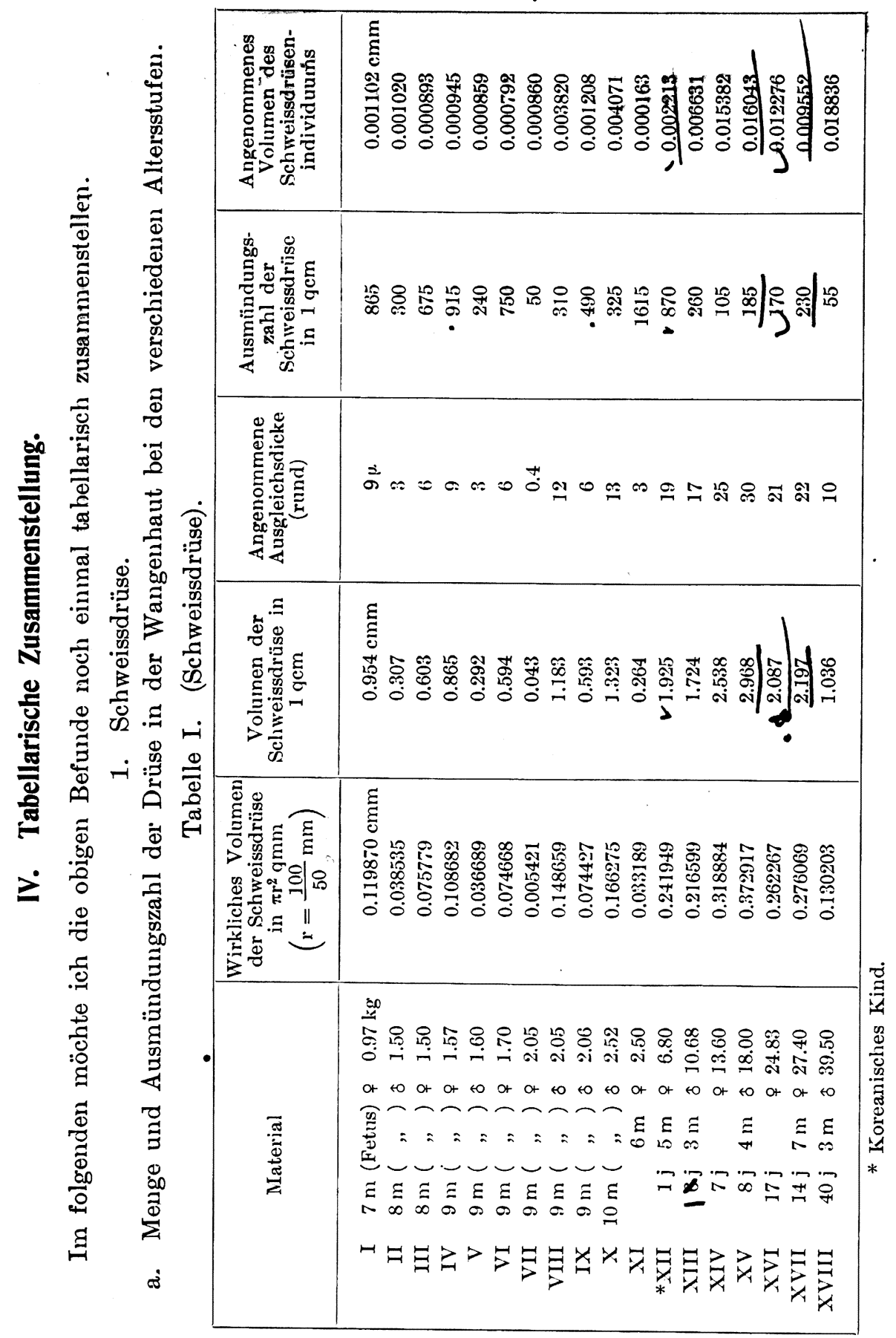




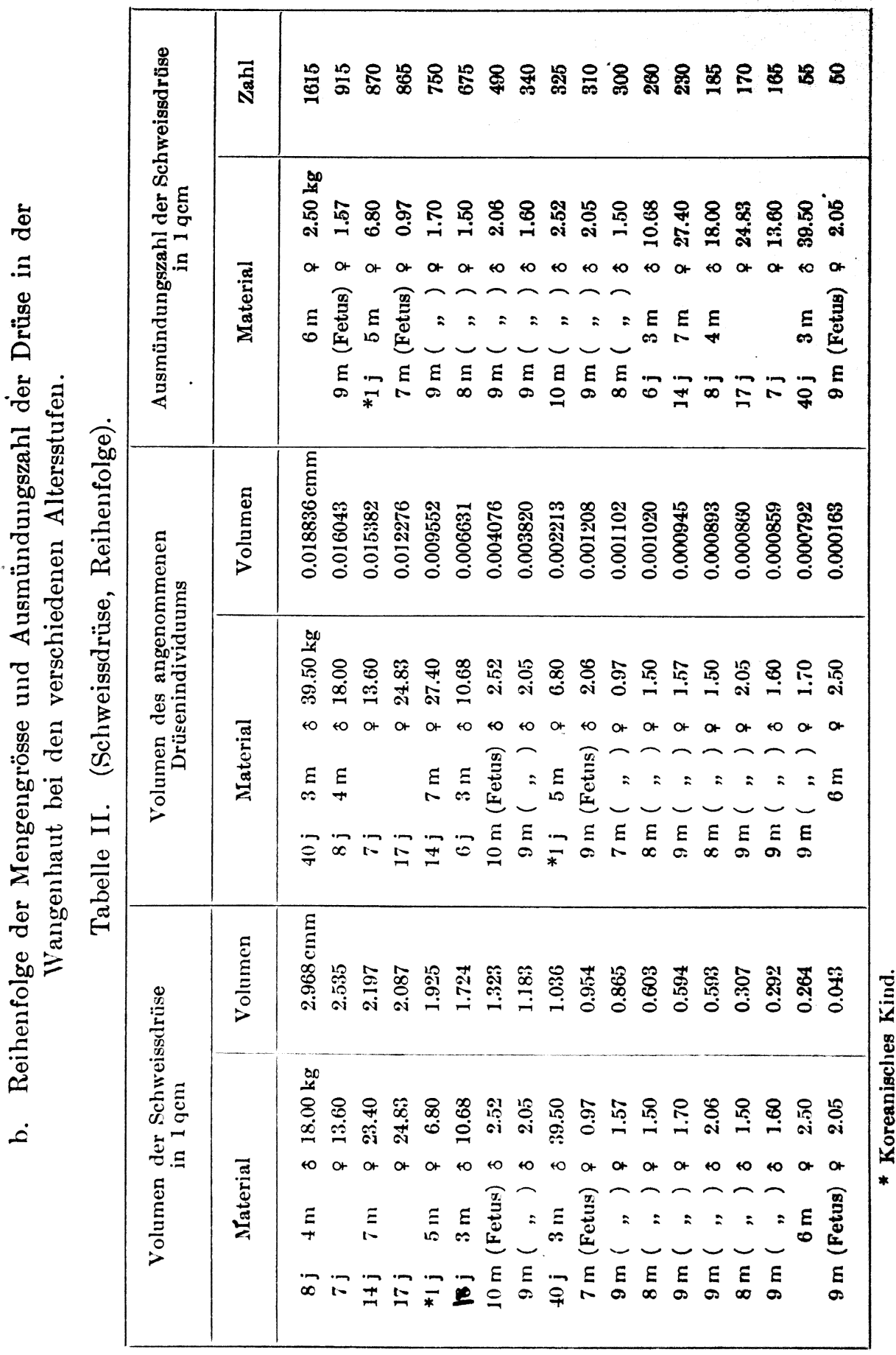


Quantitative Untersuchung der Anhangsorgane der Wangenhaut bei den Japanern.

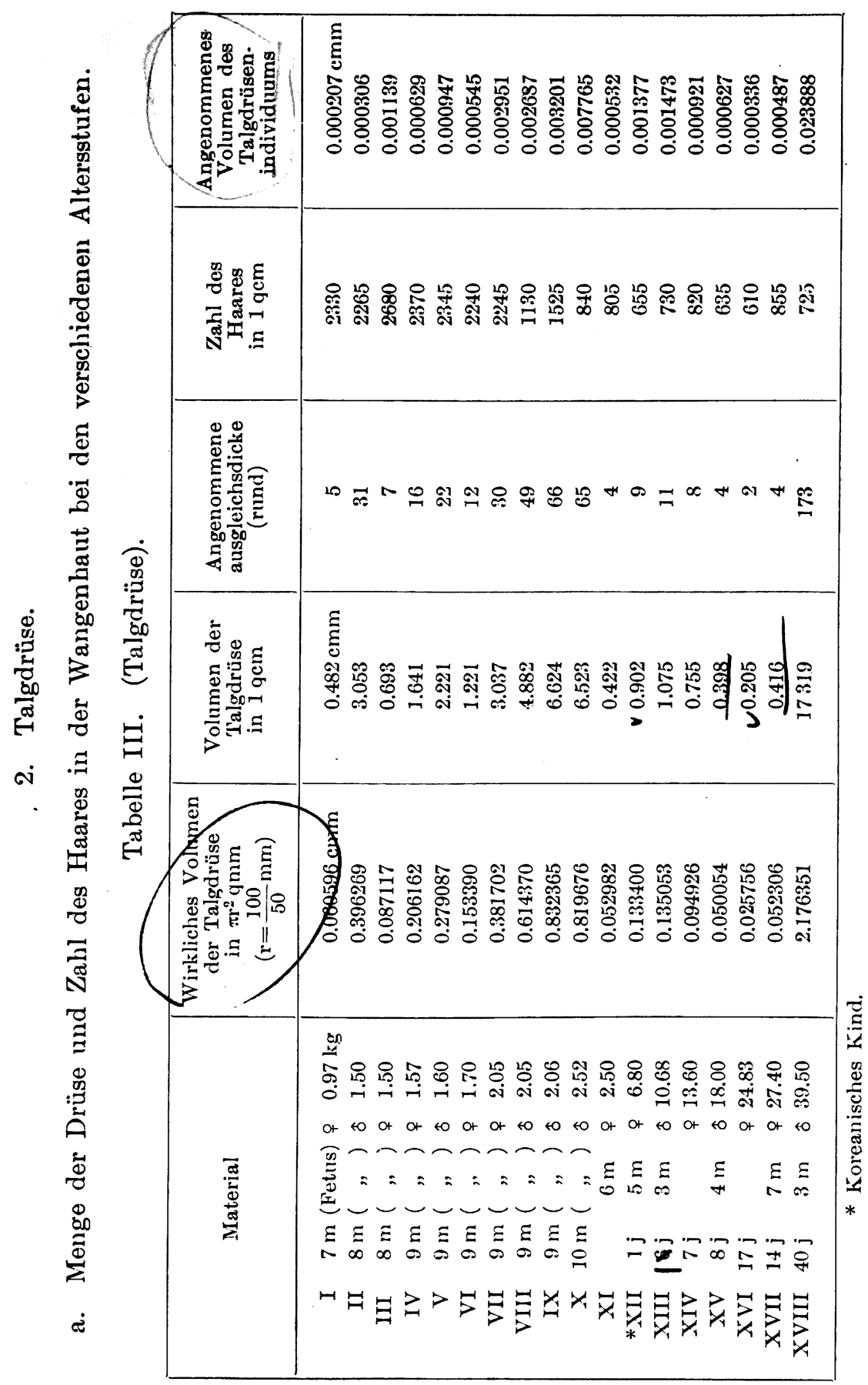




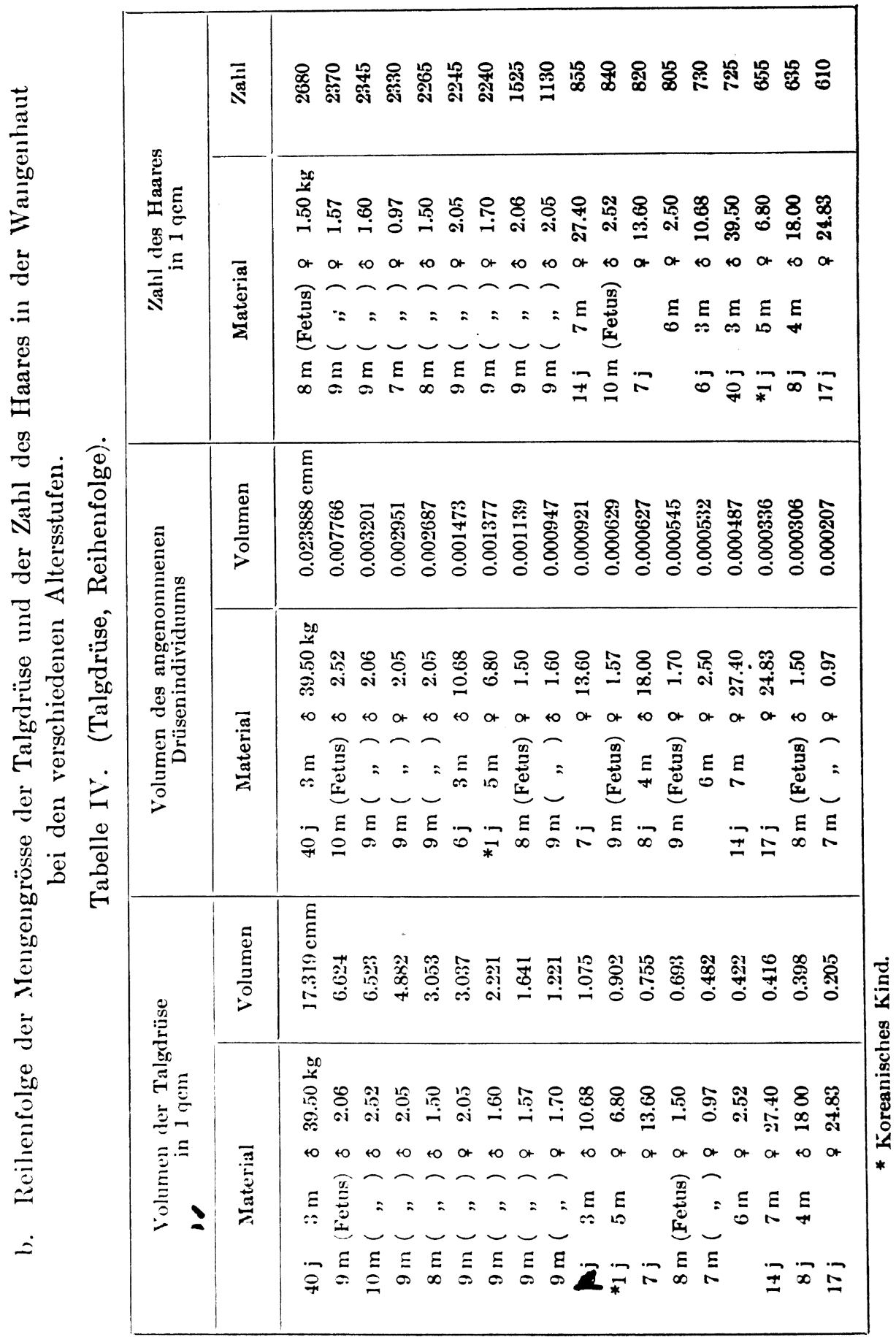


Quantitative Untersuchung der Anhangsorgane der Wangenbaut bei den Japanern.

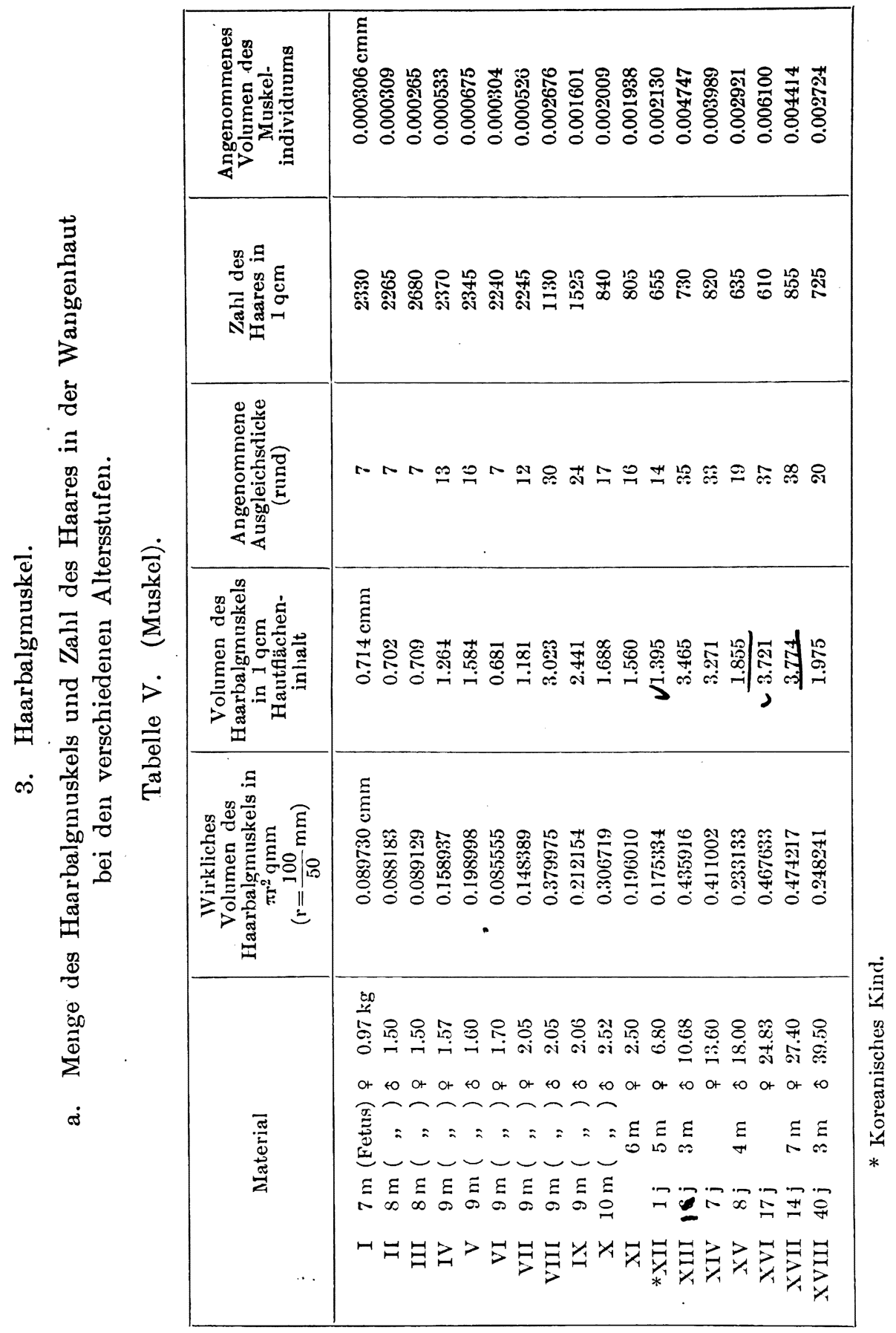




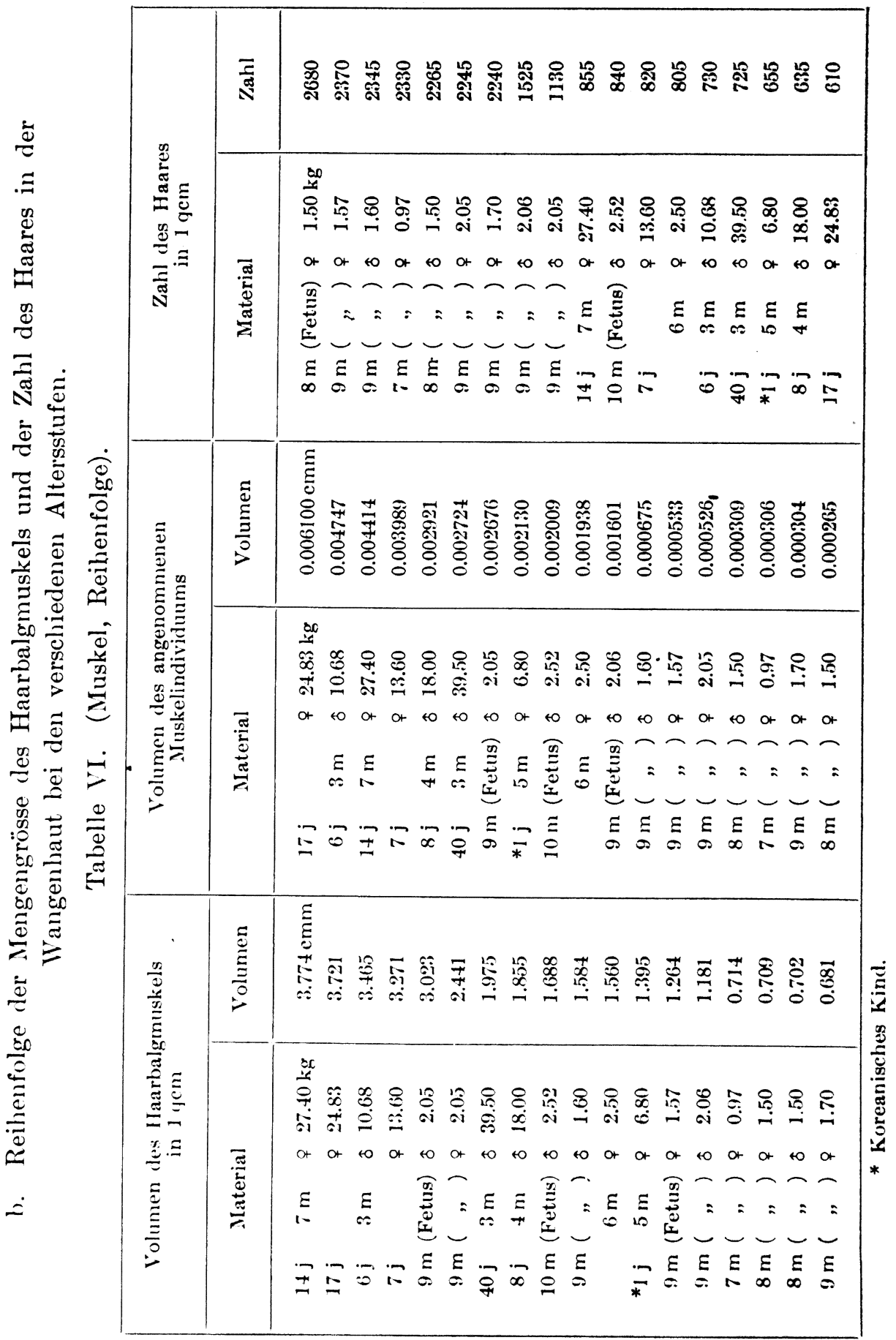


Quantitative Untersuchung der Anhangsorgane der Wangenhaut bei den Japanern.

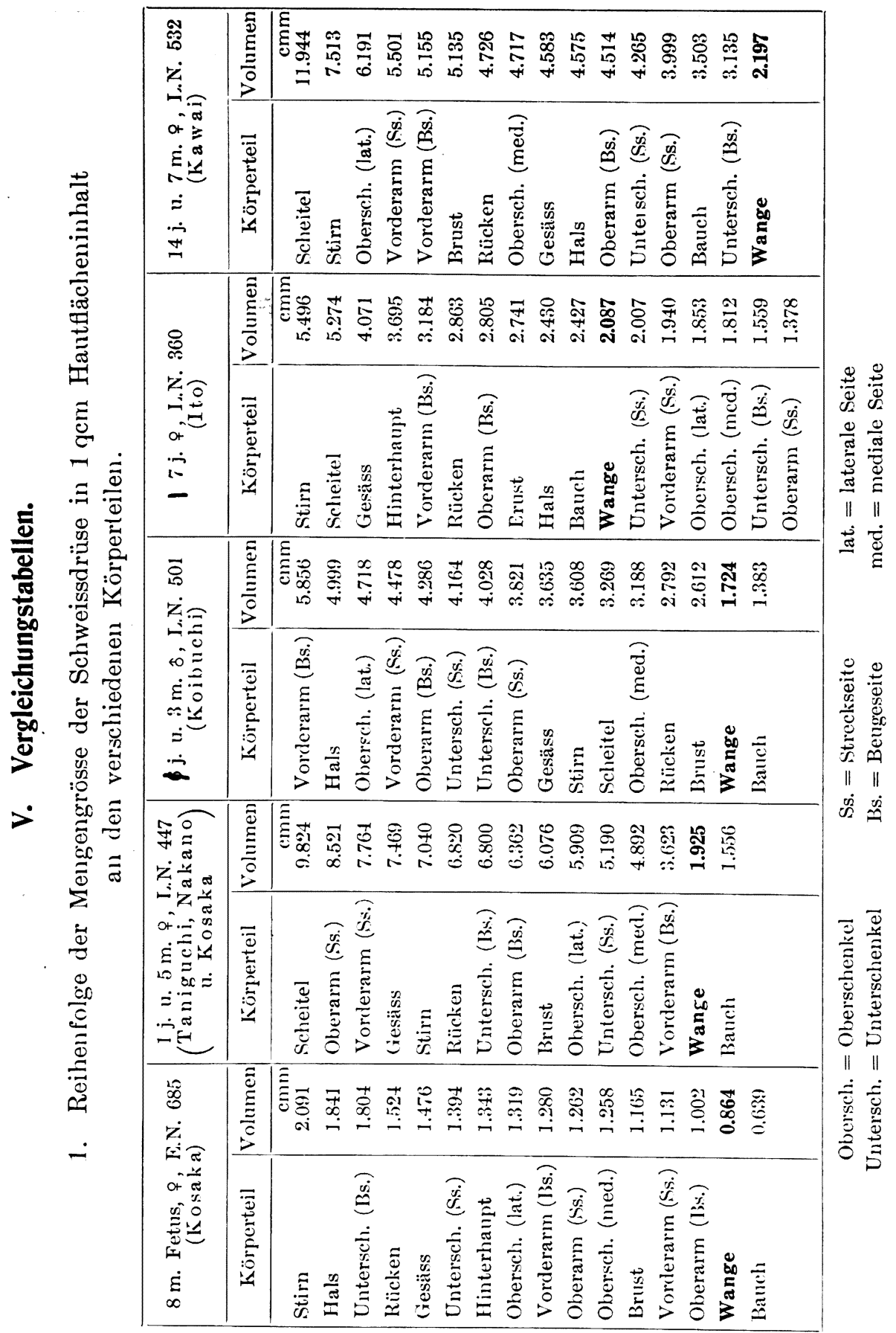




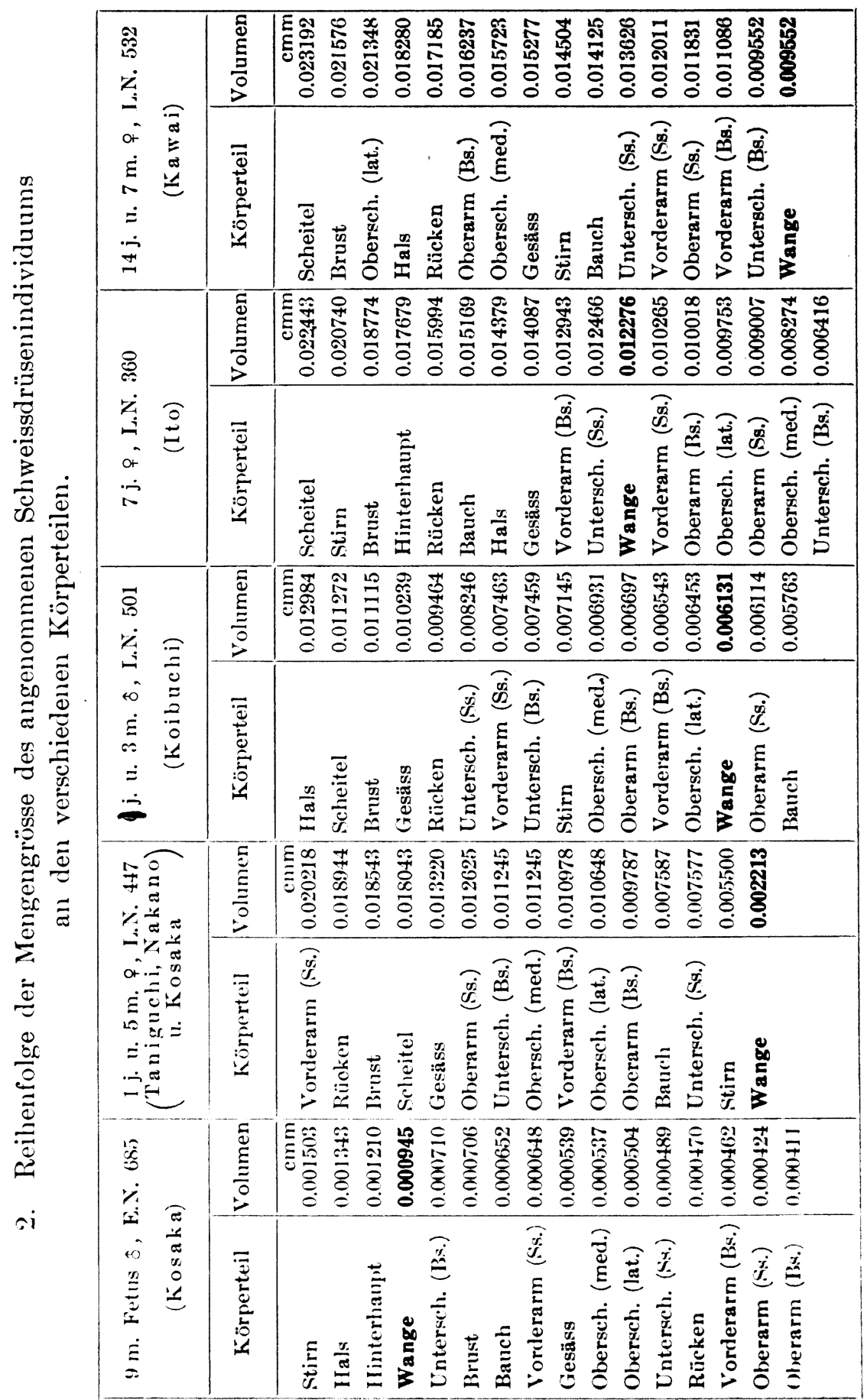


Quantitative Untersuchung der Anhangsorgane der Wangenhaut bei den Japanern. $\quad 621$

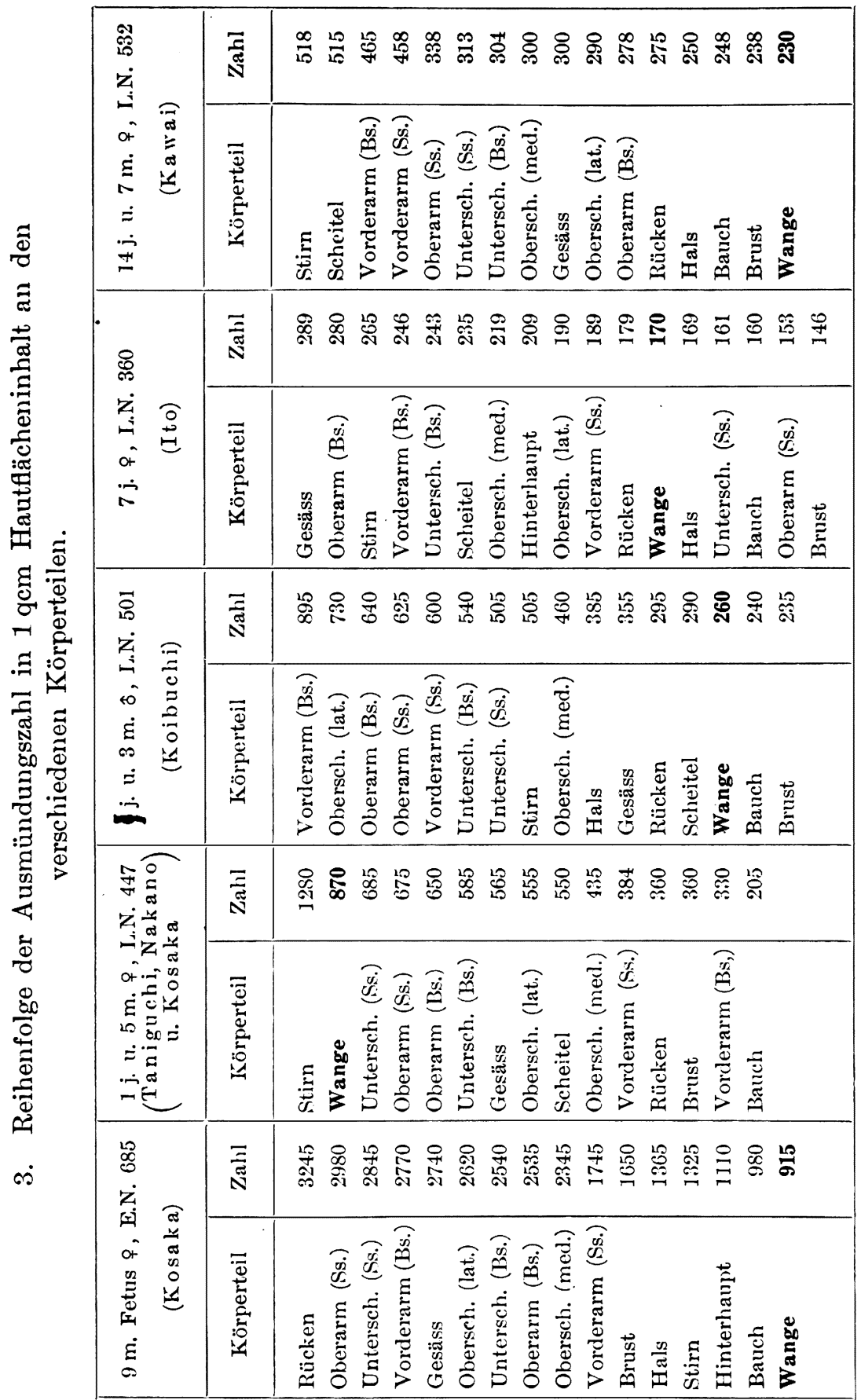




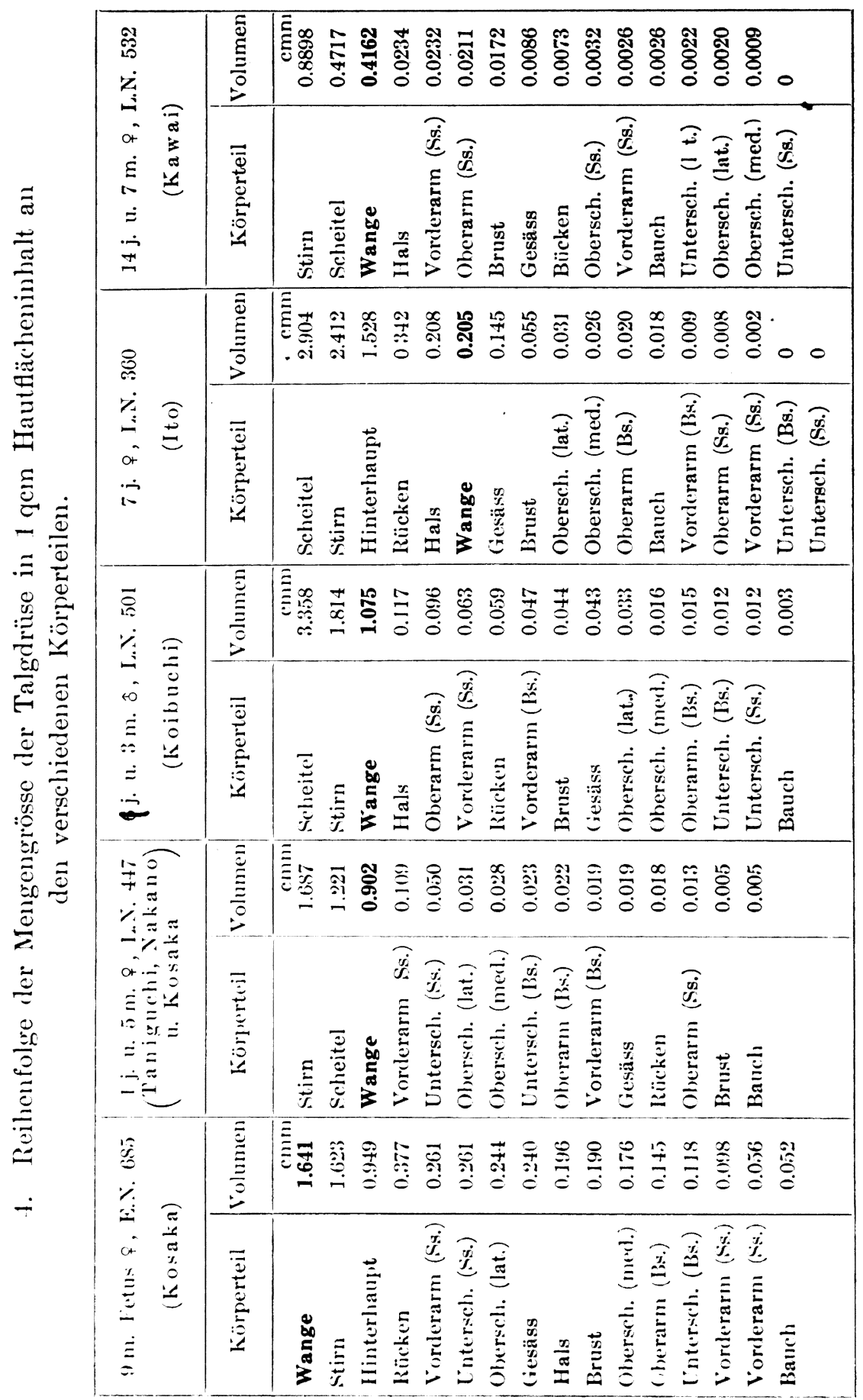


Quantitative Untersuchung der Anhangsorgane der Wangenhaut bei den Japanern. $\quad 623$

\begin{tabular}{|c|c|c|c|}
\hline \multirow{3}{*}{ 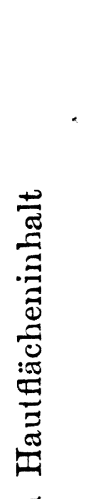 } & \multirow{2}{*}{ 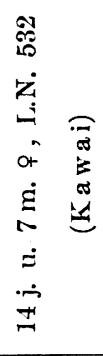 } & 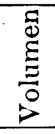 & 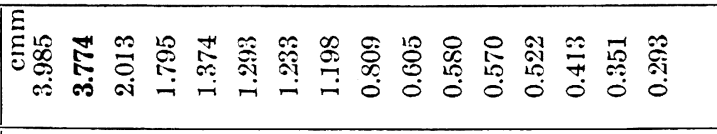 \\
\hline & & 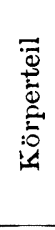 & 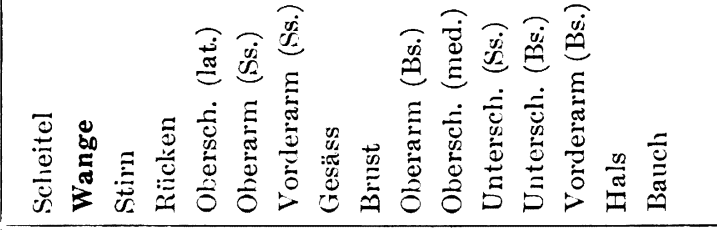 \\
\hline & \multirow{2}{*}{ 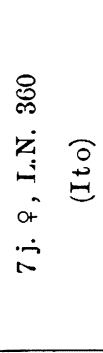 } & 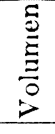 & 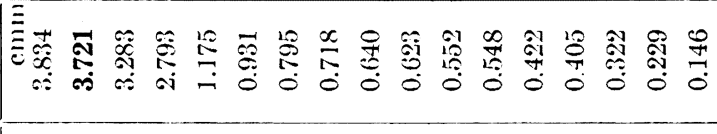 \\
\hline \multirow{4}{*}{ 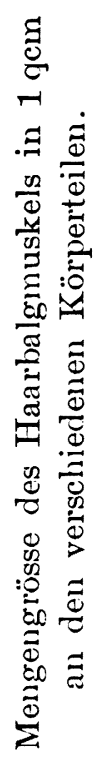 } & & 离 & 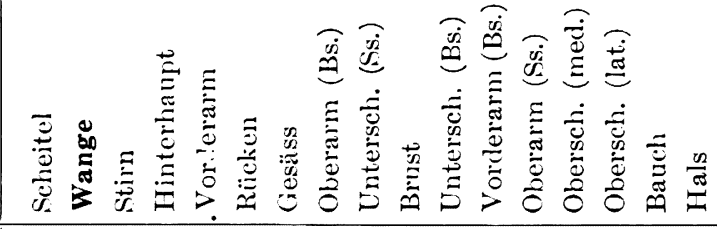 \\
\hline & \multirow{2}{*}{ 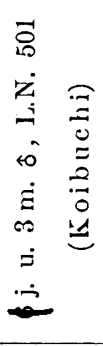 } & $\begin{array}{l}\stackrel{\bar{\Xi}}{\Xi} \\
\bar{\partial}\end{array}$ & 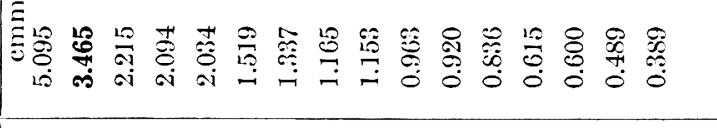 \\
\hline & & 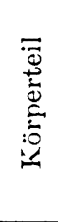 & 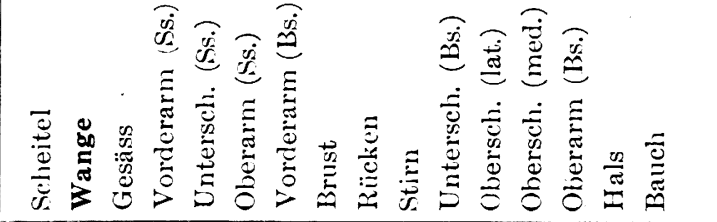 \\
\hline & \multirow{2}{*}{ 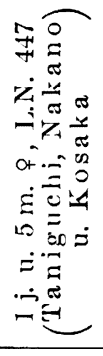 } & 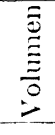 & 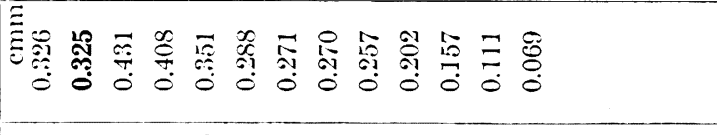 \\
\hline $\begin{array}{l}0 \\
0 \\
0 \\
0 \\
0 \\
0 \\
0\end{array}$ & & 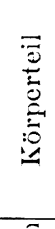 & 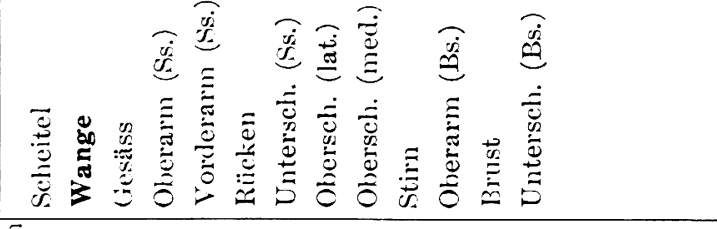 \\
\hline${ }^{\circ}$ & \multirow{2}{*}{ 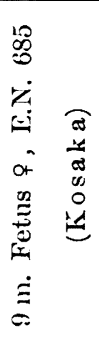 } & 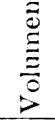 & 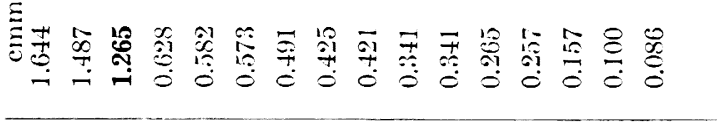 \\
\hline & & 牙 & 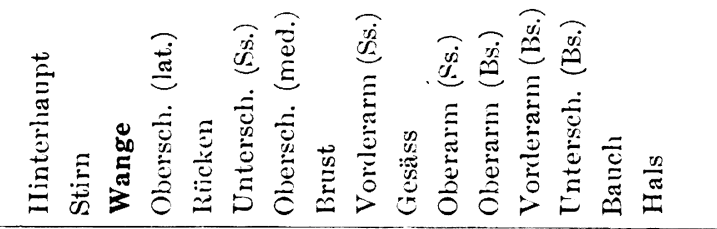 \\
\hline
\end{tabular}




\section{Zusammenfassung.}

In den obigen Schlusstabellen (Tabellen I-VI) sind die genaueren Mengenverhältnisse der Auhangsorgane der 18 untersuchten Wangenhautteile bei verschiedenen Altersstufen und Geschlechtern angegeben. Im folgenden werde ich nur die wichtigeren Befunde zusammenfassend beschreiben.

1. Das Volumen der Schweissdrüse in $1 \mathrm{qcm}$ Hautflächeninhalt der Wange variiert nach dem Alter, wie in den Tabellen I und II angegeben. Die Drüsenmenge ist beiın 8 jährigen und 4 monatlichen Knaben $(2.968 \mathrm{cmm})$ am grössten unter den 18 untersuchten Individuen, dann kommen das 7 jährige $(2.538 \mathrm{cmm})$, das $147 / 12$ jährige $(2.197 \mathrm{cmm})$, das 17 jährige $(2.087 \mathrm{cmm})$, das $11 / 2$ jährige Mädchen $(1.925 \mathrm{cmm})$, der 6 1/4 jährige Knabe $(1.724 \mathrm{cmm})$, der 10 monatliche ( $\hat{o}$ ) von 2.52 $\mathrm{kg}$ Körpergewicht $(1.323 \mathrm{cmm})$, der 9 monatliche Fetus ( $\hat{\delta})$ von $2.05 \mathrm{~kg}$ $(1.183 \mathrm{cmm})$, der $401 / 4$ jährige Mann $(1.036 \mathrm{cmm})$, der 7 monatliche (우) von $0.97 \mathrm{~kg}(0.954 \mathrm{cmm})$ der $\dot{9}$ monatliche (우) von $1.57 \mathrm{~kg}(0.865$ $\mathrm{cmm})$, der 8 monatliche ( क ) von $1.50 \mathrm{~kg}(0.603 \mathrm{cmm})$, der 9 monatliche (ㅇ ) von $1.70 \mathrm{~kg}(0.594 \mathrm{cmm})$, der 9 monatliche ( $\hat{\text { ) }})$ von $2.06 \mathrm{~kg}(0.593$ $\mathrm{cmm})$, der 8 monatliche $(\hat{\delta})$ von $1.50 \mathrm{~kg}(0.307 . \mathrm{cmm})$, der 9 monatliche Fetus ( $\hat{o}) 1.60 \mathrm{~kg}(0.292 \mathrm{cmm})$, der 6 monatliche Säugling ( o ) $(0.264$ $\mathrm{cmm}$ ); es ist am geringsten beim 9 monatlichen Fetus ( $q$ ) von $2.05 \mathrm{~kg}$ $(0.043 \mathrm{cmm})$, so dass die relative Schwankungsbreite der Drüsennnengen 68:1 beträgt.

Aus der Tabelle II erkennt man noch weiter, dass die Drüsenmenge in 1 qcm Hautflächeninhalt bei den fetalen Individuen kleiner ist als diejenige bei den postfetalen, so dass die durchschnittliche Menge bei den letzteren $(1.4739 \mathrm{cmm}) 2.1 \mathrm{mal}$ grösser als bei den ersteren $(0.6756$ $\mathrm{cmm})$ ist.

Die Zahl der Ausmündungen der Schweissdrüsen in 1 qcm Hautflächeninhalt bei verschiedenen Altern zeigt auch eine erhebliche Schwankung, so in $1 \mathrm{qcm}$ zwischen 1615 beim 6 monatlichen Säugling ( $\%$ ) und 5) beim 9 monatlichen Fetus $(f)$ von $2.05 \mathrm{~kg}$. Zwischen diesen beiden kommen der 9 monatliche Fetus ( क ) von $1.57 \mathrm{~kg}(915)$, das $11 / 2$ jährige Märchen (870), der 7 monatliche (q) von $0.97 \mathrm{~kg}$ (865), der 9 monatliche (q) von $1.70 \mathrm{~kg}$ (750), der 8 monatliche ( $q$ ) von $1.50 \mathrm{~kg}(675)$, der 9 monatliche $(\hat{o})$ von $2.06 \mathrm{~kg}(490)$, der 9 monatliche $(\hat{o})$ von 1.60 $\mathrm{kg}(340)$, der 10 monatliche ( $\hat{o})$ von $2.52 \mathrm{~kg}$ (325), der 9 monatliche ( $\hat{o})$ von $2,05 \mathrm{~kg}(310)$, der $\&$ monatliche Fetus $(\hat{o})$ von $1.50 \mathrm{~kg}(300)$, der $61 / 4$ jährige Knabe (260), das $147 / 12$ jährige Mädchen (230), der 
8 1/3 jährige Knabe (185), das 17 jährige (170), das 7 jährige Mädchen (165) und der 40 1/4 jährige Mann (55); also beträgt die relative Schwankungsbreite der Ausmündungszahl der Schweissdrüse ca. 32:1 (Tabellen I und II).

Die Ausmündungszahl der Schweissdrüse in $1 \mathrm{qcm}$ Hautflächeninhalt (Tabellen I und II) zeigt die mit dem Alter Schritt haltende Verminderung. In der Tabelle II findet man ein Zahlenverhältnis vor, nämlich, dass die Ausmündungszahl der Schweissdrüse in $1 \mathrm{qcm}$ Hautflächeninhalt bei den Feten im Mittel 562 beträgt, ist also 1.2 mal grösser als diejenige bei den postfetalen Körpern, wo sie sich durchschnittlich auf 468 in $1 \mathrm{qcm}$ beläuft.

Wie von Taniguchi zuerst vorläufig angenommen wurde, wemn eine Schweissdrüse nur eine Ausmündung besitzt, so wird das Volumen der einzelnen Schweissdrüse leicht berechnet, nämlich:

$$
\text { ( } \left.\frac{\text { Menge der Schweissdrüse in } 1 \mathrm{qcm}}{\text { Zahl der Ausmüudung in } 1 \mathrm{qcm}}\right) \text {. }
$$

Diese angenommene einzelne Schweissdrüsenmenge schwankt auch nach dem Alter erheblich, so ist sie beim $401 / 4$ jährigen Mann $(0.018836$ $\mathrm{cmm}$ ) am reichlichsten, dann folgen der $81 / 3$ jährige Knabe (0.016043 cmm), das 7 jährige $(0.015382 \mathrm{cmm})$, das 17 jährige $(0.012276 \mathrm{cmm})$, das $147 / 12$ jährige Mädchen $(0.009552 \mathrm{cmm})$, der $61 / 4$ jährige Knabe $(0.006631 \mathrm{cmm})$, der 10 monatliche ( $\hat{o})$ von $2.52 \mathrm{~kg}$ Körpergewicht $(0.004076 \mathrm{cmm})$, der 9 monatliche Fetus ( $\hat{o})$ von $2.05 \mathrm{~kg}(0.003820 \mathrm{cmm})$, das $11 / 2$ jährige Mädchen $(0.002213 \mathrm{cmm})$, der 9 monatliche $(\hat{o})$ von $2.06 \mathrm{~kg}(0.001208 \mathrm{cmm})$, der 7 monatliche (ㅇ) von $0.97 \mathrm{~kg}(0.001102$ $\mathrm{cmm})$, der 8 monatliche ( o ) von $1.50 \mathrm{~kg}(0.001020 \mathrm{cmm})$, der 9 monatliche (ㅇ ) von $1.57 \mathrm{~kg}(0.000945 \mathrm{cmm})$, der 8 monatliche ( q) $1.50 \mathrm{~kg}$ $(0.000893 \mathrm{cmm})$, der 9 monatliche $($ o $)$ von $2.05 \mathrm{~kg}(0.000860 \mathrm{cmm})$, der 9 monatliche $(q)$ von $1.60 \mathrm{~kg}(0.000859 \mathrm{cmm})$, der 9 monatliche Fetus ( क ) von $1.70 \mathrm{kgr}(0.00792 \mathrm{cmm})$, und beim 6 monatlichen Säugling ( क ) $(0.000163 \mathrm{cmm})$ ist sie am kleinsten; also beträgt die relative Schwankungsbreite ca. 116:1.

Die genaueren gegenseitigen Verhältnisse zwischen der Menge des Drüsenindividuums, der Schweissdrüsenmenge und der Zahl der Schweissdrüsenausmündungen in einem bestimmten Hautfächeninhalt sind in der Tabelle II angegeben, woraus man leicht ersehen kann, dass die Zahl der Ausmündungen in einem bestimmten Hautflächeninhalt, wie schon erwähnt, sich mit der Zunahme des Alters nach und nach vermindert, während das Volumen der einzelnen Drüsen und die gesamte 
Drüsenmenge in einem bestimmten Hautflächeninhalt, mit einiger Ausnahme, in allgeıneinen sich mit dem Alter Hand in Hand vermehren.

In den Vergleichstabellen 1, 2 und 3 findet man die obigen Befunde in der Wange mit denjenigen in den verschiedenen Körperteilen desselben Individuums verglichen. In diesen Tabellen erkennt man, dass an der Wange die Schweissdrüsenmenge in $1 \mathrm{qcm}$ Hautfächeninhalt, die Mengengrösse der angenommenen Schweissdrüsenindividuen und die Schweissdrüsenausmündungszahl in $1 \mathrm{qcm}$ Hautflächeninhalt meistens verhältnismässig kleiner als diejenigen an den anderen Körperteilen sind. Jaher kann man wohl sagen, dass die Wangenhaut relativ geringere Schweissdrüsen in Gegensatz zur Haut anderer verschiedener Körperteile hat.

2. Das Volumen der Talgdrüse auf $1 \mathrm{qcm}$ Hautflächeninhalt der Wange ist nicht gleich gross je nach dem Alter, wie in der Tabellen III und IV angegeben, woraus inan ersieht, dass die Talgdrüsenmenge in 1 qcm Hautstück in der fetalen Zeit im allgemeinen viel grösser ist als diejenige in der postfetalen mit Ausuahme der erheblichen Grösse bei einem $401 / 4$ jährigen Mann $(17.319 \mathrm{cmm})$. Dem folgen der 9 monatliche $(\hat{o})$ von $2.06 \mathrm{~kg}(6.624 \mathrm{cmm})$, der 10 monatliche ( $\hat{o}$ ) von $2.52 \mathrm{~kg}(6.523 \mathrm{cmm})$, der 9 monatliche $(\hat{o})$ von $2.05 \mathrm{~kg}(4.882 \mathrm{cmm})$, der 8 monatliche $(\hat{o})$ von $1.50 \mathrm{~kg}(3.053 \mathrm{cmm})$, der 9 monatliche (o ) von $2.05 \mathrm{kgr}(3.037 \mathrm{cmm})$, der 9 monatliche $(\hat{o})$ von $1.60 \mathrm{~kg}(2.221 \mathrm{cmm})$, der 9) monatliche ( o ) von $1.57 \mathrm{~kg}(1.641 \mathrm{cmm})$, der 9 monatliche Fetus ( o ) von $1.70 \mathrm{~kg}(1.221 \mathrm{cmm})$, der $61 / 4$ jährige Knabe $(1.075 \mathrm{cmm})$, das $15 / 12$ jährige $(0.902 \mathrm{cmm})$, das 7 jährige Mädchen $(0.775 \mathrm{cmm})$, der 8 monatliche ( $q$ ) von $1.50 \mathrm{~kg}(0.693 \mathrm{cmm})$, der 7 monatliche Fetus ( $q$ ) von $0.97 \mathrm{~kg}(0.4 \$ 2 \mathrm{cmm})$, der (; monatliche Säugling ( 9$)(0.422 \mathrm{cmm})$, das $147 / 12$ jährige Mädchen $(0.416 \mathrm{cmm})$ und der $\$ 1 / 3$ jährige Knabe $(0.398 \mathrm{cmm})$; es ist am geringsten beim 17 jährigen Mädchen $(0.205 \mathrm{cmm})$, so dass die relative Schwankungsbreite $85: 1$ beträgt. Dieser Wert ist ganz ähnlich demselben der Schweissdrüse.

J)ie Haarzahl in $1 \mathrm{gcm}$ Hautfächeninhalt (Tabellen III und V) schwankt auch nach den Altersstufen erheblich, so zwischen 2680 beim 8 monatlichen Fetus ( $q$ ) von $1.50 \mathrm{~kg}$ und 610 beim 17 jährigen Mädchen; zwischen diesen beiden kommen der 9 monatliche ( q ) von $1.57 \mathrm{~kg}$ (2370), der $)$ monatliche ( 3 ) von $60 \mathrm{~kg}(2345)$, der 7 monatliche ( $q$ ) von 0.97

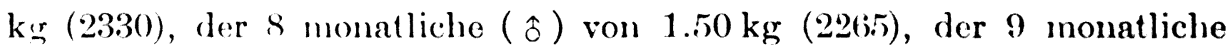
(q) von $2.05 \mathrm{~kg}(2215)$, der 9 monatliche ( $q$ ) von $1.70 \mathrm{~kg}$ (2240), der 9 monatliche $(j)$ von $2.06 \mathrm{~kg}(1525)$, der 9 monatliche lietus ( $\hat{o}$ ) von $2.05 \mathrm{~kg}$ (1130), das $147 / 12$ jährige Mädchen (555), der 10 momatliche 
Fetus ( $\hat{o}$ ) von $2.52 \mathrm{~kg}$ (840), das 7 jährige Mädchen (820), der 6 monatliche Säugling (ㅇ) (805), der $61 / 4$ jährige Knabe (730), der $401 / 4$ jährige Mann (725), das 1 1/2 jährige Mädchen (655), und der 8 1/3 jälırige Knabe (635); also die relative Schwankungsbreite 4.3:1 beträgt. Daraus erkennt man, dass die im hestimmten Hautstück vorkommende Haarzahl sich mit dem Alter nach und nach vermindert.

In den meisten Fällen meiner Schnittpräparate ist die mehrhaarige Haargruppe schwer zu unterscheiden; fast alle Haare befinden sich als einhaarig verstreut.

Ninmt man nun vorläufig an, dass jede Talgdrüse einem Haare gehört, so wird das Volumen der einzelnen Talgdrüse leicht berechnet:

$$
\left(\frac{\text { Menge der Talgdrüse in } 1 \mathrm{gcm}}{\text { Zahl des Haares in } 1 \mathrm{qcm}}\right) \text {. }
$$

Das Volumen dieser angenommenen einzelnen Talgdrüse ist auch nach den Altersstufen verschieden 'gross, so beim $401 / 4$ jährigen Mann $(0.023888 \mathrm{cmm})$ am grössten, damn folgen der 10 monatliche ( $\hat{o})$ von $2.52 \mathrm{~kg}(0.007765 \mathrm{cmm})$, der 9 monatliche $(\hat{o})$ von $2.06 \mathrm{~kg}(0.003201$ cmm), der 9 monatliche ( ㅇ ) von $2.05 \mathrm{~kg}(0.002951 \mathrm{cmm})$, der 9 monatliche Fetus $(\hat{o}) 2.05 \mathrm{~kg}(0.002687 \mathrm{cmm})$, der $61 / 4$ jährige Knabe $(0.001473 \mathrm{cmm})$, das $11 / 2$ jährige Mädchen $(0.001377 \mathrm{cmm})$, der $\mathrm{S}$ monatliche $(₹)$ von $1.50 \mathrm{~kg}(0.001139 \mathrm{cmm})$, der 9 monatliche Fetus $(\hat{o})$ von $1.60 \mathrm{~kg}(0.000947 \mathrm{cmm})$, das 7 jährige Mädchen $(0.000921 \mathrm{cmm})$, der 9 monatliche Fetus ( 9 ) von $1.57 \mathrm{~kg}(0.000629 \mathrm{cmm})$, der $81 / 3$ jährige Knabe $(0.000627 \mathrm{cmm})$, der 9 monatliche Fetus ( क ) von $1.70 \mathrm{~kg}(0.000545$ $\mathrm{cmm})$, der 6 monatliche Säugling ( ( ) $(0.000532 \mathrm{cmm})$, das $147 / 12$ jährige $(0.000487 \mathrm{cmm}), 17$ jährige Mädchen $(0.000336 \mathrm{cmm})$ und der 8 monatliche Fetus ( $\hat{o})$ von $1.50 \mathrm{~kg}(0.000306 \mathrm{cmm})$, und es ist am kleinsten beim 7 monatlichen Fetus (ㅇ ) von $0.97 \mathrm{~kg}(0.000207 \mathrm{cmm})$. So beläuft sich die relative Schwankungsbreite des Volumens der mit einem Haare zusammenhängenden Talgdrüse auf ca. 115:1. Diese Mengengrösse der einzelnen Talgdrüse mit einem Haare ist ganz unabhängig vom Alter, also zwischen der gesanten Drüsenmenge in $1 \mathrm{gcm}$ Hautstück und dem Volumen des Drüsenindividuums kann man kein bestinmtes Verhältnis ersehen.

In der Vergleichstabelle 4 sind die obigen Befunde mit anderen Körperregionen bei den verschiedenen Altersstufen verglichen dargestellt, woraus man ersehen kamn, dass die Talgdrüsenmenge in einem bestimmten Hautflächeninhalt in der Wangenhaut eine der Stirn oder den behaarten Kopfteilen nachfolgende grössere Menge zeigt. Deshalb kann man sagen, 
dass die Wangenhaut, unter den verschiedenen Körperteilen, verhältnisinässig reichlichere Talgdrüsen hat, und deren Mengengrösse derselben der Kopfhaut nahe steht.

3. Das Volumen des Haarbalgmuskels in 1 qcm Hautfächeninhalt der Wange (Tabellen V und VI) ist beim 14 7/12 jährigen Mädchen $(3.774 \mathrm{cmm})$ am grössten, daun folgen das 17 jäbrige Mädchen (3.721 $\mathrm{cmm}$ ), der $61 / 4$ jährige Knabe (3.465 cmm), 7 jähriges Mädchen (3.271 cmın), der 9 monatliche ( $\hat{\delta})$ von $2.05 \mathrm{~kg}(3.023 \mathrm{cmm})$, der 9 monatliche Fetus ( ㅇ ) von $2.06 \mathrm{~kg}(2.441 \mathrm{cmm})$, der $401 / 4$ jährige.Mann $(1.975 \mathrm{cmm})$, der 8 1/3 jährige Knabe $(1.855 \mathrm{cmm})$, der 10 monatliche ( $\hat{o})$ von 2.52 $\mathrm{kg}(1.688 \mathrm{cmm})$, der 9 monatliche Fetus ( o ) von $1.60 \mathrm{~kg}(1.584 \mathrm{cmm})$, der 6 mỏnatliche Säugling ( 9 ) $(1.560 \mathrm{cmm})$, das 1 1/2 jährige Mädchen $(1.395 \mathrm{cmm})$, der 9 monatliche $($ o $)$ von $1.57 \mathrm{~kg}(1.264 \mathrm{cmm})$, der 9 monatliche $(\hat{o})$ von $2.06 \mathrm{~kg}(1.181 \mathrm{cmm})$, der 7 monatliche ( $q$ ) von $0.97 \mathrm{~kg}(0.714 \mathrm{cmm})$, der 8 monatliche (ㅇ ) von $1,50 \mathrm{~kg}(0.709 \mathrm{cmm})$, der 8 inonatliche Fetus ( $\hat{o})$ von $1.50 \mathrm{~kg}(0.702 \mathrm{cmm})$; es ist am geringsten beim 9 monatlichen Fetus $(\uparrow)$ von $1.70 \mathrm{~kg}(0.681 \mathrm{cmm})$. Die relative Schwankungsbreite beträgt also $6: 1$.

Die Mengengrösse des Haarbalgmuskels in einem bestimmten Hautflächeninhalt ist nicht gleich gross je nach dem Alter, wie in der Tabelle $\mathrm{V}$ gezeigt. Die Muskelmenge in $1 \mathrm{qcm}$ Hautflächeninhalt bei den fetalen Individuen, ist im umgekehrten Verhältnisse zu der Talgdrüsenmenge in demselben Hautteil kleiner als bei den postfetalen.

Die Menge des einzelnen Muskels in verschiedenen Altersstufen, der mit einem Haare im Zusammenhang steht, wurde in derselben Weise wie beim Talgdrüsenindividuum berechnet:

$$
\left(\frac{\text { Menge des Muskels in } 1 \mathrm{qcm}}{\text { /ahl des Haares in } 1 \mathrm{qcm}}\right) \text {. }
$$

Also ergibt es sich aus der Tabelle VI, dass das Volumen des einzelnen Muskels, der zu einem Haare gehört, beim 17 jährigen Mädchen $(0.006100 \mathrm{cmm})$ ain grössten ist, danach folgen der $61 / 4$ jährige Kuabe $(0.004747 \mathrm{cmm})$, das $147 / 12$ jährige $(0.004114 \mathrm{cmm})$, das 7 jährige Mädchen (0.003989 cmm), der $81 / 3$ jährige Knabe ( $\hat{o})(0.002921 \mathrm{cmm})$, der 40 $1 / 3$ jährige Mann $(0.002724 \mathrm{cmm})$, der 9 monatliche Fetus $(\hat{o})$ von $2.0 .5 \mathrm{~kg}(0.002627 \mathrm{cmm})$, das $11 / 2$ jährige Mädchen $(0.002130 \mathrm{cmm})$, der 10 monatliche Fetus $(\hat{o})$ von $2.52 \mathrm{~kg}(0.002009 \mathrm{cmm})$, der 6 monatliche Säugling ( $q$ ) $(0.001938 \mathrm{cmm})$, der 9 monatliche $(\hat{o})$ von $2.06 \mathrm{~kg}$ $(0.001601 \mathrm{cmm})$, der 9 monatliche Fetus $(\hat{\delta})$ von $1.60 \mathrm{~kg}(0.00675 \mathrm{cmm})$, der 9 monatliche ( $q$ ) von $1.57 \mathrm{~kg}(0.000533 \mathrm{cmm})$, der 9 monatliche ( $q$ ) 
von $2.05 \mathrm{~kg}(0.000526 \mathrm{cmm})$, der 8 monatliche ( $\hat{)})$ von $1.50 \mathrm{~kg}(0.000309$ cinm) und der 9 monatliche Fetus ( $\%$ ) von $1.70 \mathrm{~kg}(0.000301 \mathrm{cmm})$; es ist am kleinsten beim 8 monatlichen Fetus ( $\%$ ) von $1.50 \mathrm{~kg}(0.000265$ cmin). Der relative Grenzwert beträgt daher 23:1.

Die Menge des zu einem Haare gehörenden Haarbalgmuskels ist bei all den Feten, mit Ausnahme des 9 monatlichen Fetus ( $\beta$ ) von $2.05 \mathrm{~kg}$ kleiner als bei den postfetalen Individuen. Die gesamte Mengengrösse des Muskels im bestimmten Hautflächeninhalt parallerisiert im grossen gauzen mit dem Alter.

In Bezug auf die Vergleichung der obigen Befunde mit denen der anderen verschiedenen Körperregionen wird auf die Vergleichstabelle 5 hingewiesen. Daraus ergibt sich, dass die Muskelmenge in $1 \mathrm{gcm}$ Hautflächeninhalt an der Wange, wie bei der Talgdrïse, im Gegensatz zu anderen Körperregionen, fast immer ziemlich gross ist. Daher kamn man sagen, dass unter den verschiedenen Körperteilen, wie die obigen Tabellen angeben, an der Wange der Haarbalgmuskel inmer sehr reichlich vorhanden ist und seine Dichtigkeit hier derjenigen des Kopfes am nächsten steht.

Zum Schluss danke ich Herrn Professor K. Okajima aufs herzlichste für seine stete liebenswürdige Unterstützung bei dieser Arbeit.

\section{Literatur.}

1) Okajima u. Kanaizuka: Quantitative Untersuchung des Haarbalgmuskels bei den Saïgetieren. Folia Anat. Japon. Bd. VII. 1929.

2) Taniguchi: Quantitative Untersuchung der Anhangsorgane der Haut bei den japanischen Neugeborenen. Folia Anat. Japon. Bd. IX. 1931.

3) Koibuchi: Quantitative Untersuchung der Anhangsorgane der Haut bei dem japanischen Kind. Folia Anat. Japon. Bd. X. 1932.

4) Yamada: Quantitative Untersuchung der Anhangsorgane der Haut bei dem Deutschen. Folia Anat. Japon. Bd. X. 1932.

5) Kosaka u. Taniguchi: Quantitative Untersuchung der Anhangsorgane der Haut bei einem japanischen Fetus. Folia Anat. Japon. Bd. X. 1932.

6) Taniguchi, Kosaka u. Nakano: Quantitative Untersuchung der Anhangsorgane der Haut bei einem koreanischen Kind. Folia Anat. Japon. Bd. XI. 1933.

7) Kawai: Quantitative Untersuchung der Anhangsorgane der Haut bei einem erwachsenen Ainu. Folia Anat. Japon. Bd. XI. 1933.

8) Kawai: Quantitative Untersuchung der Anhangsorgane der Haut bei einem japanischen Mädchen. Folia Anat. Japon. Bd. XI. 1933. 
9) Kawaji: Quantitative Untersuchung der Anhangsorgane der Haut bei dem Finne. Folia Anat. Japon. Bd. XII. 1934.

10) Ka wa ji: Quantitative Untersuchung der Anhangsorgane der Haut des Handrūckens bei den Japanern. Folia Anat. Japon. Bd. XII. 1934.

11) Ito: Quantitative Untersuchung der Anhangsorgane der Haut bei einer japanischen Frau. Folia Anat. Japon. Bd. XII. 1934.

12) Taniguchi: Quantitative Untersuchung der Anhangsorgane der Haut bei einem Hydrokephalus. Folia Anat. Japon. Bd. XIII. 1935.

13) Shibasama: Quantitative Untersuchung der Anhangsorgane der Haut bei dem Bantu-Xosa. Folia Anat. Japon. Bd. XIII. 1935.

14) Taniguchi: Über die Verteilung der Schweissdrüsen beim Menschen. Nagora Iji Shinshi. Bd. 8. 1934 (Japanisch).

15) Taniguchi: Quantitative Untersuchung der Anhangsorgane der Haut bei einem Bantu-M'gonie. Folia Anat. Japon. Bd. XIII. 1935. 